\title{
tunnel routier du Fréjus : \\ les mesures géotechniques effectuées \\ sur le chantier français et leur application \\ pour la détermination \\ et l'adaptation du soutènement provisoire
}

\author{
par \\ J.-R. Beau \\ Ingénieur en Chef des Ponts et Chaussées \\ Conseiller Technique de la S.F.T.R.F. \\ Président du Comité des Experts \\ J. Cabanius \\ Président de SOFRELEC \\ Membre du Comité d'Experts \\ G. Courtecuisse \\ Ingénieur en Chef S.G.E. TPI \\ Directeur des Travaux du Groupement d'Entreprises \\ D. Fourmaintraux \\ Assistant au Laboratoire Central des Ponts et Chaussées (L.C.P.C.) \\ P. Gesta \\ Directeur Scientifique S. G. E. TPI \\ Membre du Comité d'Experts \\ M. Levy \\ Directeur à SETEC TP \\ Directeur des Travaux du Tunnel du Fréjus \\ C. Néraud \\ Directeur de SETEC TP \\ Chef du projet du Tunnel du Fréjus \\ Membre du Comité d'Experts \\ M. Panet \\ Chef du Département Géotechnique du L.C.P.C. \\ Membre du Comité d'Experts \\ J. Péra \\ Directeur du CETU \\ Membre du Comité d'Experts \\ E. Tincelin \\ Professeur à l'École des Mines de Paris \\ Membre du Comité d'Experts \\ G. Vouille \\ Laboratoire de Mécanique des Roches de l'École des Mines de Paris \\ Membre suppléant du Comité d'Experts
}

\section{Introduction}

Le tunnel routier du Fréjus est un tunnel franco-italien construit en association par deux Sociétés d'Économie Mixte : la S.F.T.R.F., Maître d'Ouvrage pour la moitié de l'ouvrage située en France et la SITAP, Maître d'Ouvrage pour la moitié située en Italie.
La S.F.T.R.F. ou Société Française du Tunnel Routier du Fréjus émane des Collectivités locales de la région Rhône-Alpes.

Le Maitre d'CEuvre de la partie française est la SETEC TRAVAUX PUBLICS. Le Groupement d'Entreprises chargé de l'exécution du génie civil au tunnel 
proprement dit, dans la zone des schistes lustrés à forte couverture, a été piloté par la Société Générale d'Entreprises (S. G. E.-TPI) et comprend des Entreprises des Groupes Fougerolle et S.C.R.E. G.

Les mesures géotechniques qui vont être présentées dans cet article, après avoir été exposées le 4 octobre 1979 devant le Comité Français de Mécanique des Roches ont été, pour la plupart, décidées par un Comité d'Experts Techniques que les trois partenaires cités plus haut ont institué d'un commun accord.

Ce Comité comprenait :

- un représentant du Maître d'Ouvrage : M. Beau;

- un représentant du Maître d'CEuvre : M. Néraud;

- un représentant de l'Entreprise : M. Gesta;

- quatre Experts: MM. Tincelin (et M. Vouille son suppléant), Panet, Cabanius et Péra.

II était chargé de suivre les problèmes techniques posés par l'excavation, le soutènement et le revêtement et de faire des recommandations au Maître d'Ouvrage.
S'appuyant sur un très gros travail de mesures et de constatations faites par le Maître d'CEuvre avec la collaboration de l'Entreprise et l'assistance du L.C.P.C., du laboratoire de l'École des Mines et du C.E.T.E. de Lyon, il a parfaitement atteint les objectifs recherchés :

- assurer en toutes circonstances la sécurité des personnes travaillant sur le chantier; jamais en effet le soutènement provisoire ne fut mis en défaut;

- rechercher les meilleures solutions à tous les problèmes spécifiques d'excavation et de soutènement; ce résultat fut atteint dans les meilleures conditions puisque les délais d'exécution et le budget furent respectés; l'avancement moyen s'établissant à 163 mètres par mois avec un maximum de $276 \mathrm{~m}$ soit $11,5 \mathrm{~m}$ par jour;

- tirer un maximum d'enseignements d'ordre général et scientifique de ce chantier exceptionnel.

L'article qui suit, est destiné essentiellement à développer ce dernier point.

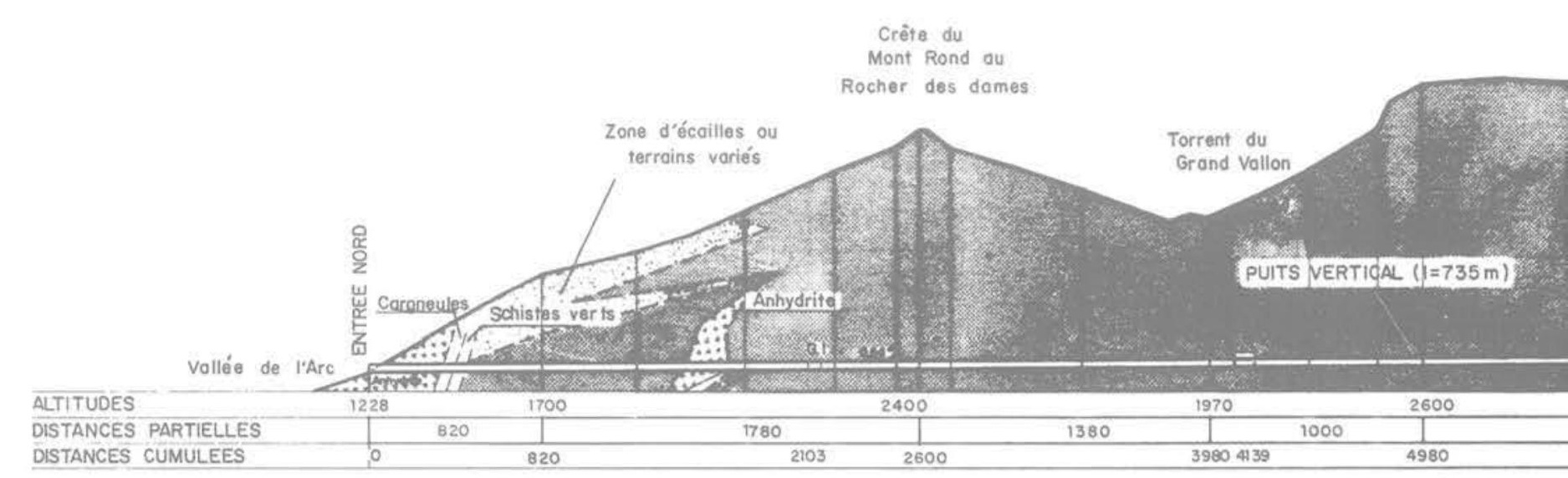

Fig. 2 Profil en long.

Fig. 3 Tracé en plan

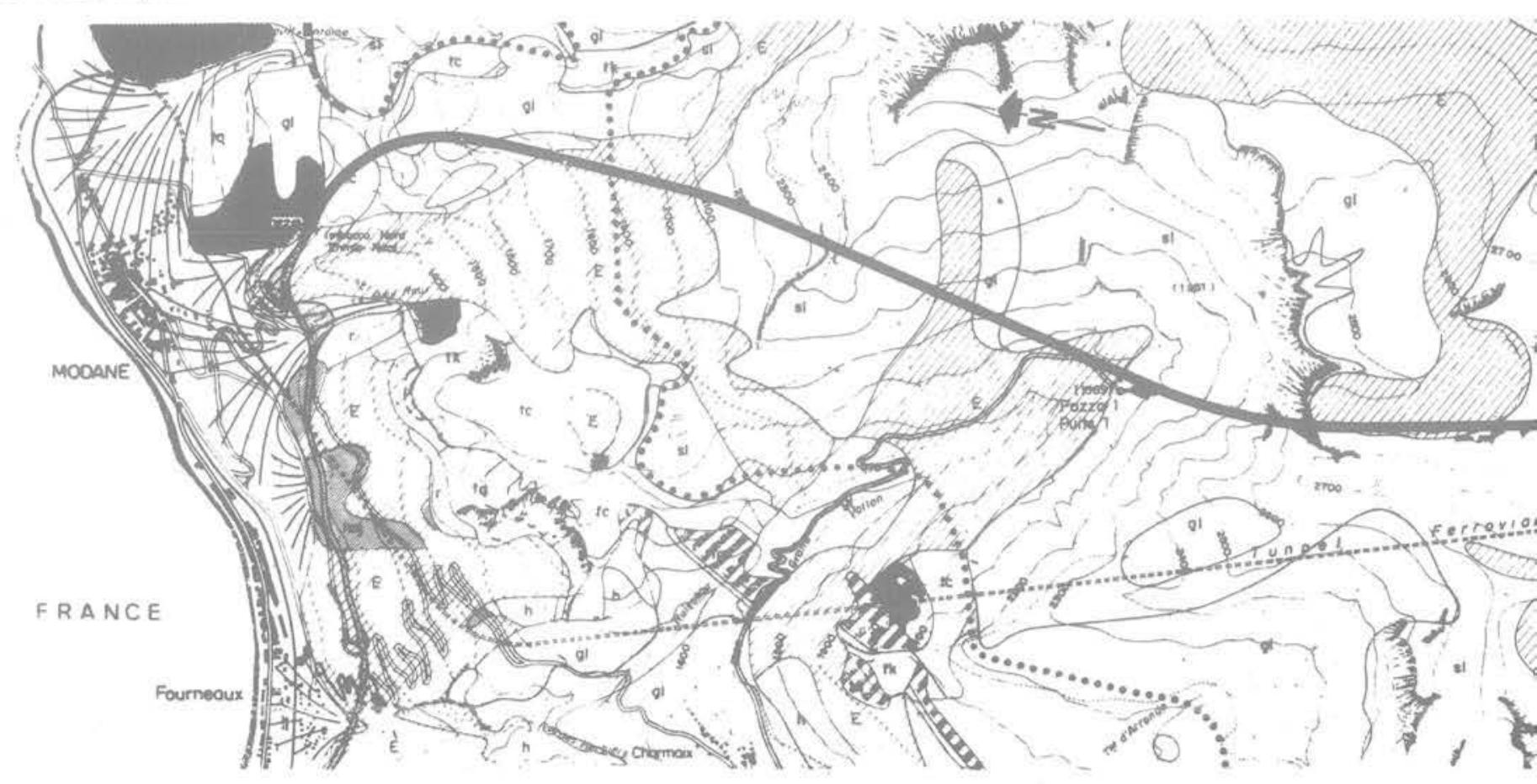




\subsubsection{Profil en long}

Le profil en long est donné par la figure 2; la pente unique de $0,54 \%$ est montante de la France vers I'Italie. Cette disposition assez exceptionnelle a pu être adoptée en raison des faibles venues d'eau prévues, qui permettaient de prendre le risque d'une longue attaque descendante. Cela a permis d'avoir une pente plus faible et plus favorable au trafic routier que si l'on avait eu un profil classique en toit. La tête française est à la cote $1228 \mathrm{~m}$; la tête italienne est à $1297 \mathrm{~m}$. La couverture de terrain naturel au-dessus du tunnel atteint $1800 \mathrm{~m}$ à la crête de la pointe du Fréjus; son profil se prête à la construction de deux puits de ventilation situés au $1 / 3$ et aux $2 / 3$ du parcours.

\subsection{Terrains rencontrés}

En dehors de la zone d'entrée de 448 m constituée de terrains variés (anhydrite, cargneules, schistes verts) et d'une zone particulière d'anhydrite compacte entre les PM 1482 et 1710 , l'ensemble des terrains rencontrés, aussi bien dans le tunnel que dans les puits, a été constitué de schistes lustrés dont le plan de schistosité, plus ou moins parallèle à la direction générale Nord-Sud du tunnel, a un pendage variable incliné en général de 25 à $50^{\circ}$ sur l'horizontale.

\subsection{Coupe en travers}

(Section courante et section en garage.)

La largeur entre piédroits est de $10,52 \mathrm{~m}$. La largeur roulable de 9,00 m entre bute-roues permet de circuler sur deux voies de 3,50 m et de maintenir les deux sens de circulation en cas de panne d'un véhicule. Le haut de la section étant utilisé pour la ventilation, la hauteur sous plafond est de $4,50 \mathrm{~m}$.

On rencontre, à l'espacement de $2 \mathrm{~km}$ environ, cinq garages avec élargissement de $2 \mathrm{~m}$ et galerie transversale pour demi-tour (fig. 4 et 5 ).

\subsection{Schéma de principe de la ventilation définitive}

Le tunnel est divisé en 6 sections de ventilation de $2140 \mathrm{~m}$ de longueur moyenne, alimentées par 6 usines ( 2 usines extérieures et 2 usines souterraines doubles, reliées avec l'extérieur par des puits).

Chaque usine comporte deux ventilateurs d'air frais de débit unitaire $127,5 \mathrm{~m}^{3} / \mathrm{s}$ et deux ventilateurs d'air vicié de débit unitaire $104 \mathrm{~m}^{3} / \mathrm{s}$.

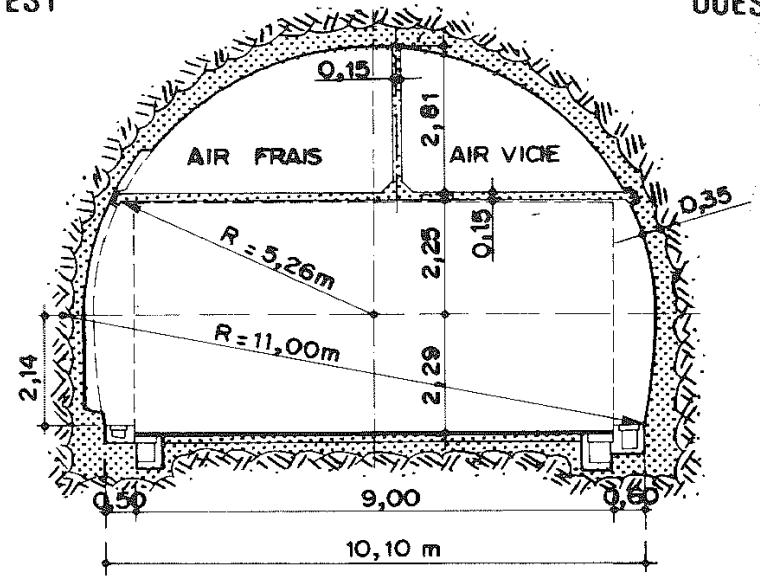

Fig. 4 Coupe en travers

Le renouvellement de l'air, rendu nécessaire par le trafic routier est obtenu en introduisant par un conduit du plafond, de l'air frais qui est réparti, tout le long du tunnel, par des bouches réglables espacées de $4,50 \mathrm{~m}$ : I'air vicié s'écoule le long du tunnel et sort par les têtes d'extrémité. Lorsque la vitesse de l'air dans le tunnel atteint $8 \mathrm{~m} / \mathrm{s}$, on aspire l'air vicié complémentaire par l'autre conduit du plafond. Ce seuil correspond à un trafic horaire d'environ 900 UVP. La ventilation permet d'admettre un trafic horaire maximum de 1800 UVP (dont $5 \%$ de poids lourds). Le circuit d'air vicié est également utilisé pour aspirer les fumées et les gaz toxiques en cas d'incendie (fig. 6).

\subsection{Les puits de ventilation}

Le puits, côté France, a un diamètre de $7,40 \mathrm{~m}$ et est divisé en deux conduits de 19 et $23 \mathrm{~m}^{2}$ par une cloison en béton armé. Il comporte une partie verticale de $700 \mathrm{~m}$ de hauteur.

Le puits, côté Italie, est constitué de deux tubes parallèles de $5,10 \mathrm{~m}$ de diamètre chacun. Ils sont inclinés à $45^{\circ}$ environ et ont une longueur de $700 \mathrm{~m}$ environ (fig. 7).

\subsection{Usines souterraines de ventilation}

Au pied de chaque puits, on trouve une usine souterraine double. L'usine, côté France, comporte deux galeries de grande section, parallèles au tunnel : la salle des ventilateurs (ou Usine) et la galerie de distribution d'air (ou galerie de pied de puits). Des galeries transversales de petite section (ou rameaux)

OUEST

Fig. 5 Coupe en travers au droit d'un garage

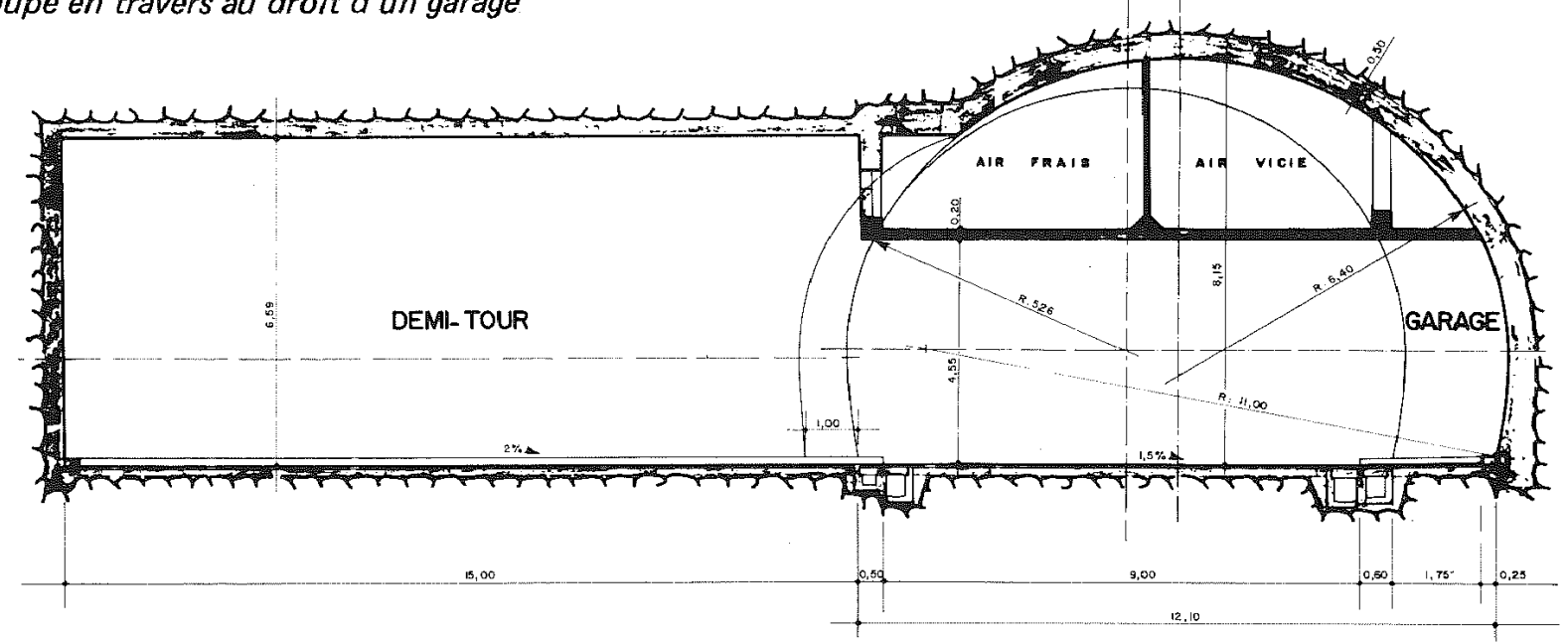




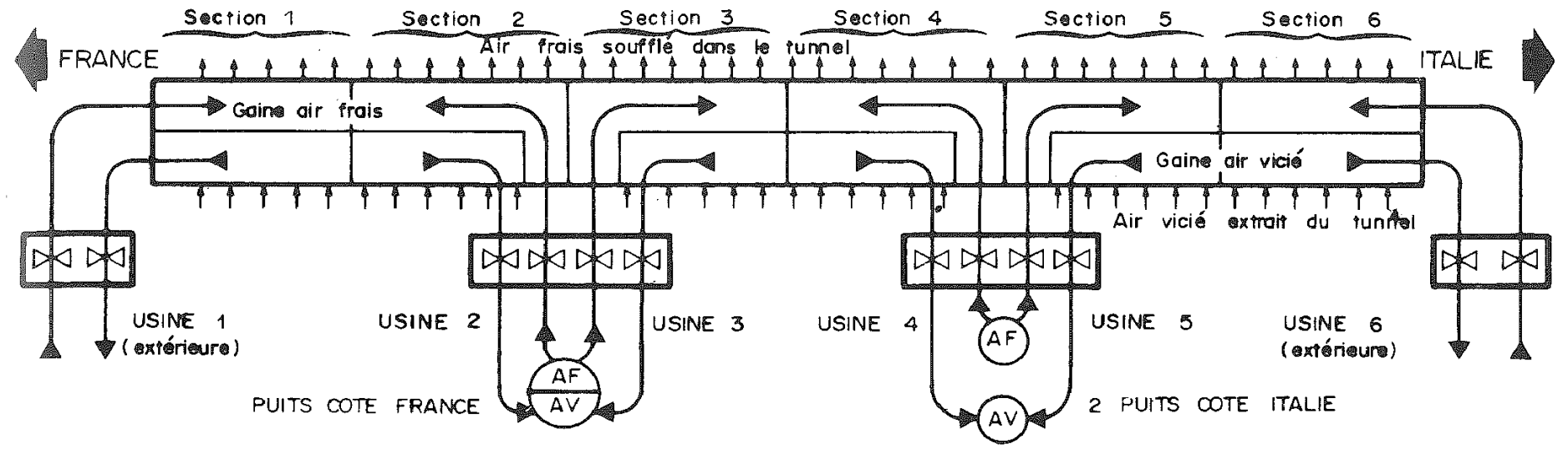

Fig. 6 Schéma de la ventilation du tunnel

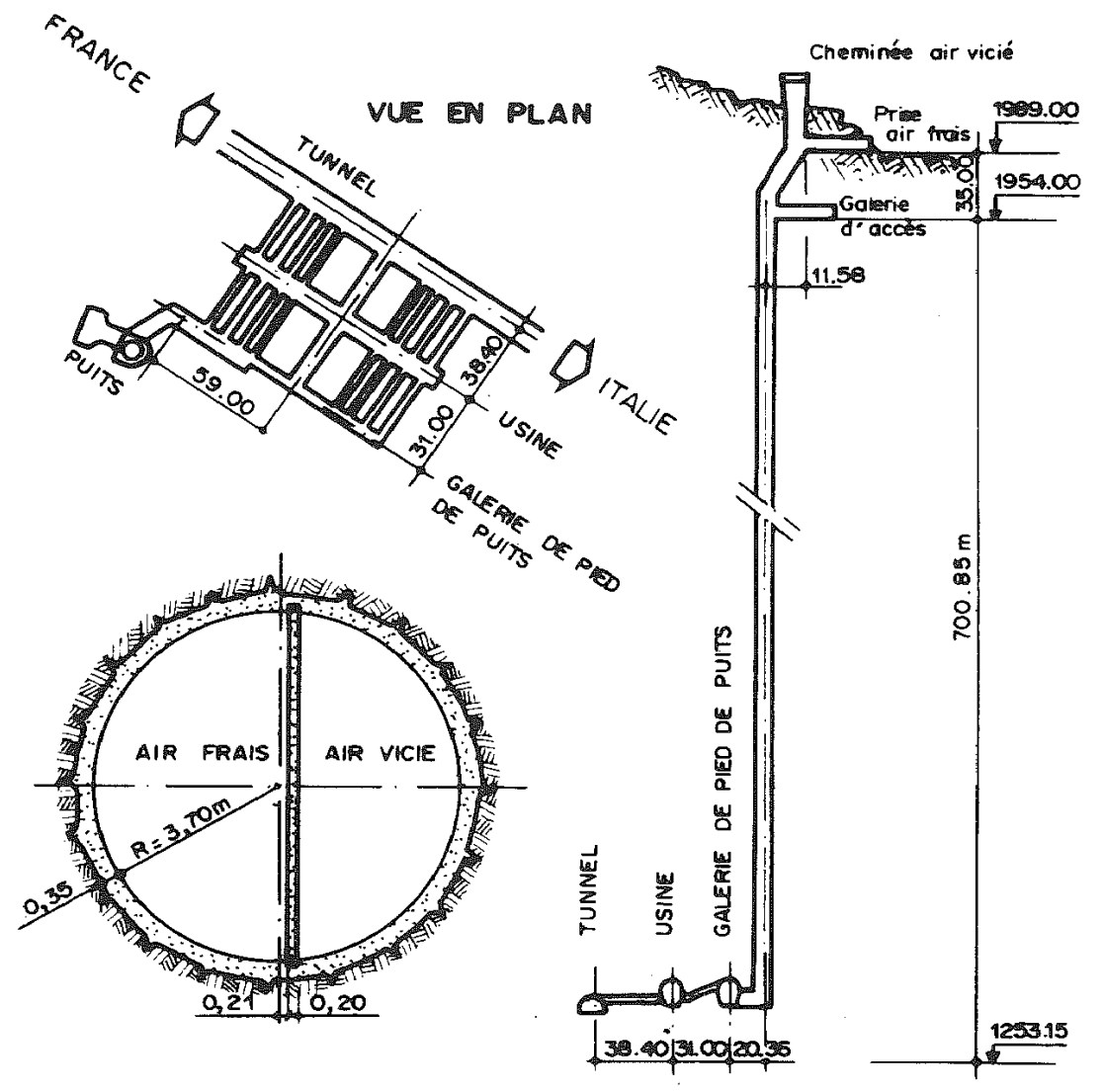

COUPE TRANSVERSALE COUPE LONGITUDIMALE

Fig. 7 Puits de ventilation

permettent à l'air, venant par le puits et la galerie de distribution d'air, de passer dans les ventilateurs, puis de gagner le plafond du tunnel et vice-versa. Une galerie demi-tour, transversale au tunnel, sert d'accès routier au centre de l'usine et se prolonge jusqu'à la galerie de distribution d'air. Enfin, deux galeries de chantier reliant directement le tunnel au pied du puits et à l'extrémité de l'Usine complètent cet ensemble (fig. $8 a, b, c)$.

\section{Description des schistes lustrés traversés}

2.1 Données géologiques et structurales sur le massif rocheux traverse

\subsubsection{Nature géologique du massif}

Le massif des schistes lustrés dans lequel est situé le Tunnel Routier du Fréjus, représente la couverture sédimentaire de la «zone piémontaise» de l'arc alpin dont le socle cristallin est le massif du Grand Paradis et de la Dora Maïra, situé en Italie bien à l'Est de Modane. Cette "couverture» vraisemblablement épaisse de plusieurs centaines voire milliers de mètres, est à sa base de nature gypseuse et calcaire et devient rapidement marneuse et marno-calcaire.

Au début de l'ère tertiaire au cours des mouvements de mise en place de la chaîne alpine, cet ensemble a été poussé en "glissant » sur sa semelle de gypses, vers l'Ouest, de l'Italie vers la France, où il s'est étalé en une vaste et épaisse série de «nappes». Démantelée ensuite par l'érosion, cette série subsiste pour son fragment occidental le plus important, à l'Ouest du massif d'Ambin entre Modane au Nord, Bardonnèche au Sud et le Col du Fréjus à l'Ouest, zone dans laquelle les tunnels ferroviaire et routier la traversent du Nord au Sud (fig. 9).

Un métamorphisme léger a «lustré » ces formations marneuses en y développant des minéraux micacés. Ces mouvements ainsi brièvement et simplement décrits ont bien entendu fortement marqué le massif. 

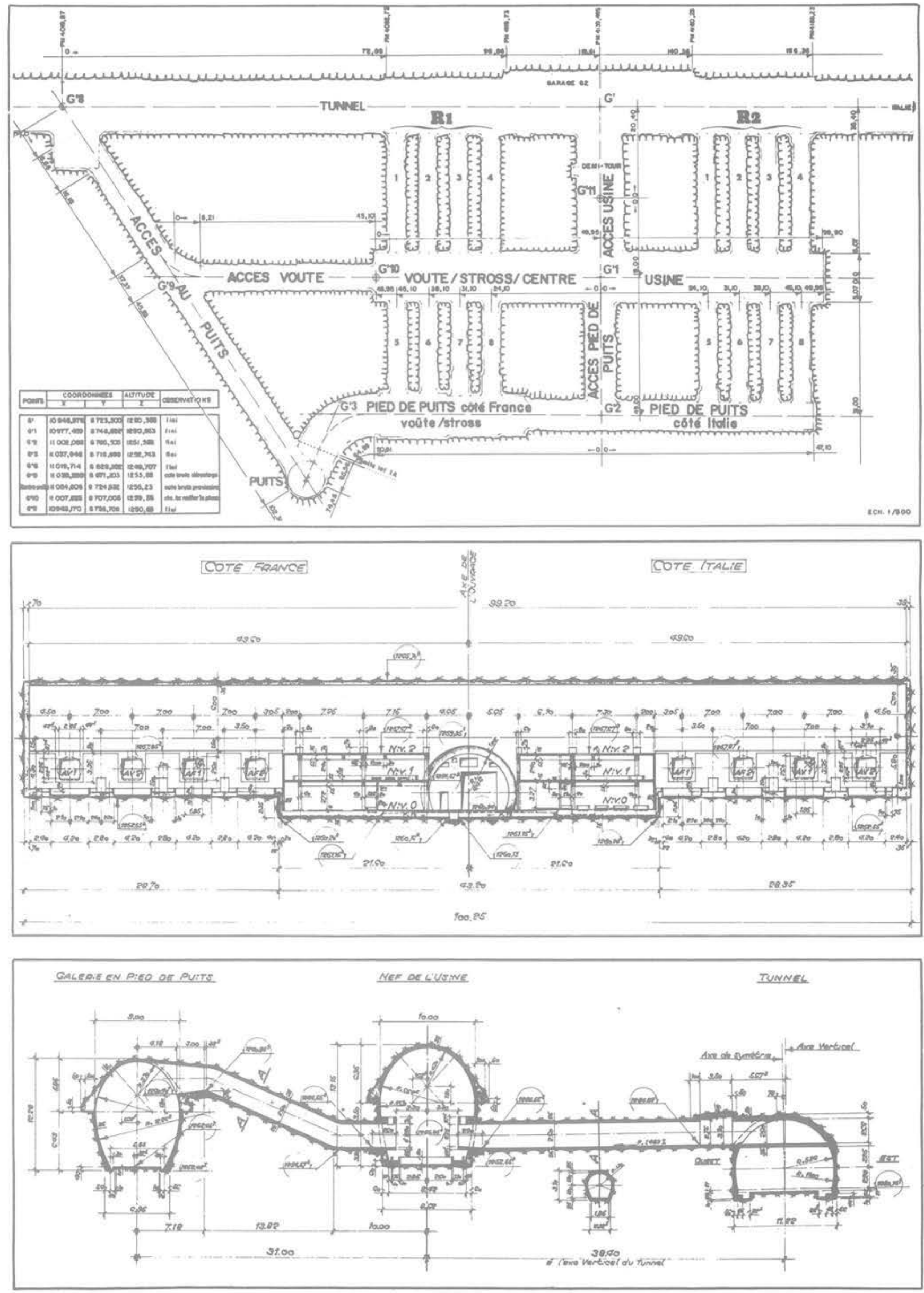

Fig. 8 Usine souterraine côté France

$8 a$ : vue en plan $-8 b:$ coupe longitudinale $-8 c$ : coupe transversale 


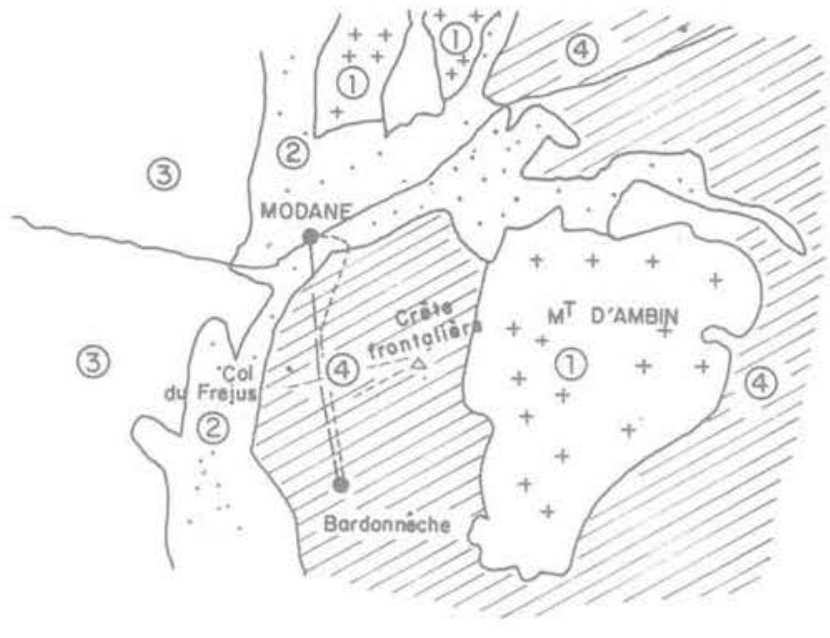

Fig. 9 Géologie du site

\subsubsection{Structure du massif}

\section{(I) Structure principale}

La structure principale est une schistosité fort marquée, développée en liaison avec les contraintes subies; son plan est grossièrement parallèle à l'axe du tunnel et son pendage varie le long de l'ouvrage entre $25^{\circ}$ et $50^{\circ}$ vers l'Ouest, atteignant localement à la faveur des rebroussements des valeurs extrêmes à $0^{\circ}$ et $70^{\circ}$; on observe un débit en bancs ou lamelles d'épaisseur généralement faible (décimétrique à centimétrique); le creusement se fait "en direction " par rapport aux plans de schistosité.

\section{(II) Autres discontinuités}

D'autres discontinuités ont été rencontrées, s'organisant en trois autres familles principales en plus de la schistosité (fig. 10) :

- une famille de diaclases $\left(45^{\circ} \mathrm{N} 180^{\circ}\right)$ perpendiculaire au plan de symétrie du tunnel (plan vertical contenant l'axe du tunnel) et dont le pendage de $45^{\circ}$ en moyenne est orienté vers le Sud:

- une famille de fractures de cisaillement important $\left(45^{\circ} \mathrm{N} 10^{\circ}\right)$, elle aussi perpendiculaire au plan de symétrie du tunnel, mais à pendage orienté vers le Nord:

- une direction de cisaillement moins marqué $\left(50^{\circ}\right.$ à $70^{\circ} \mathrm{N} 90^{\circ}$ ) à laquelle sont souvent liés des rebroussements et ondulations de la schistosité, orientée parallèlement au tunnel mais au pendage marqué vers I'Est, grossièrement orthogonal à la schistosité.

L'occurrence simultanée de certaines de ces discontinuités a entrainé localement quelques instabilités (fig.11).

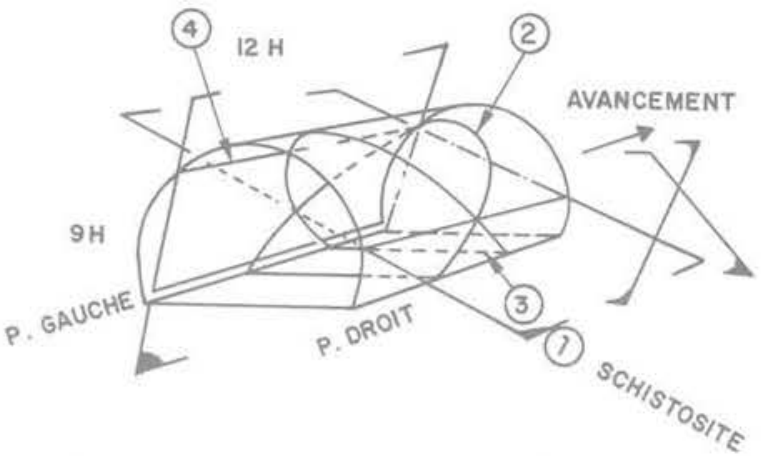

Fig. 10 Système de discontinuités affectant le massif

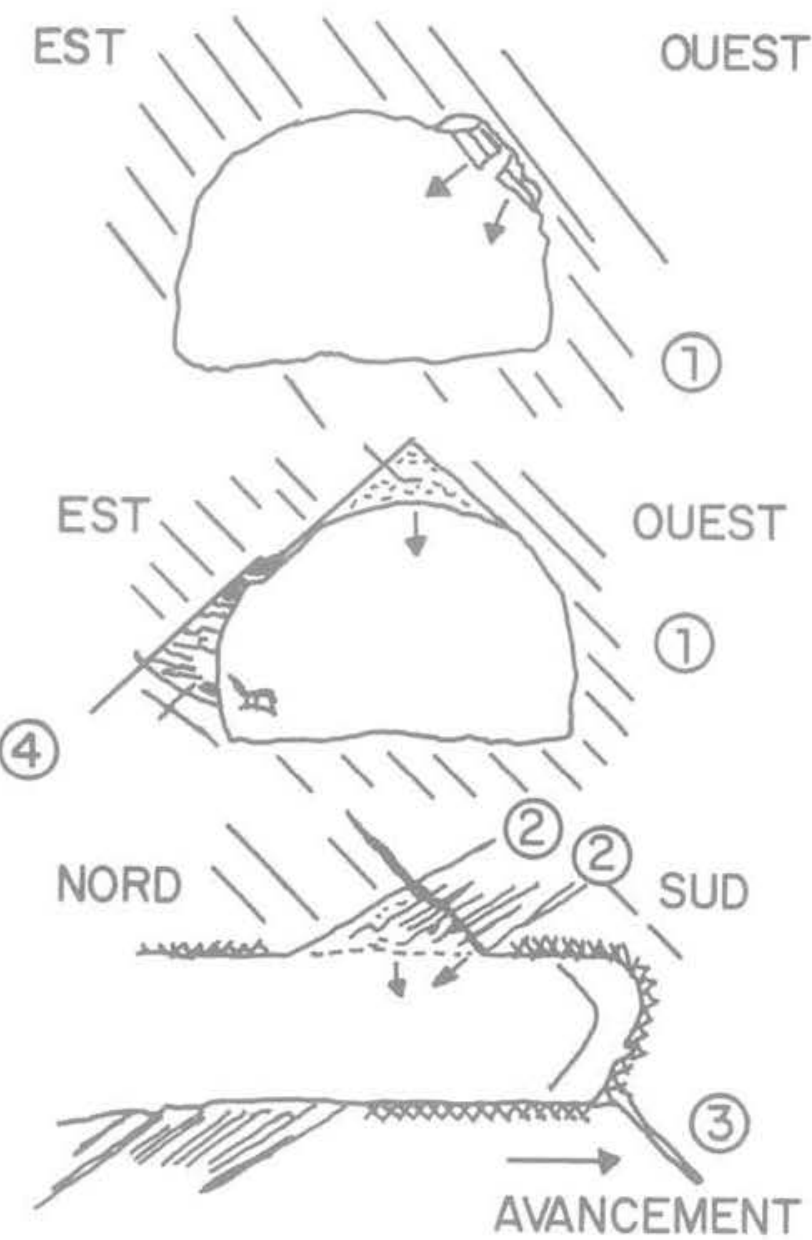

Fig. 11 Quelques instabilités liées aux discontinuités du massif

Les discontinuités apparaissant à la paroi de la galerie ont fait l'objet d'un lever systématique au 1/200 par le L. C.P. C. tout au long de l'ouvrage (fig. 12), à partir du PM 1750, où le tunnel routier pénètre dans la masse de cette nappe des schistes lustrés.

\subsection{Pétrographie et minéralogie des roches}

\subsubsection{Pétrographie}

Cette formation constituée d'une roche à texture schisteuse de teinte gris sombre à aspect luisant et au toucher doux laissant des traces gris noir, est souvent striée de filons et filonnets blancs de calcite mêlée de quartz, concordants ou discordants avec la schistosité; étirés, ils apparaissent comme des alignements lenticulaires aplatis (fig. 13).

Le débit de la roche en dalles et lamelles est plus ou moins aisé. 


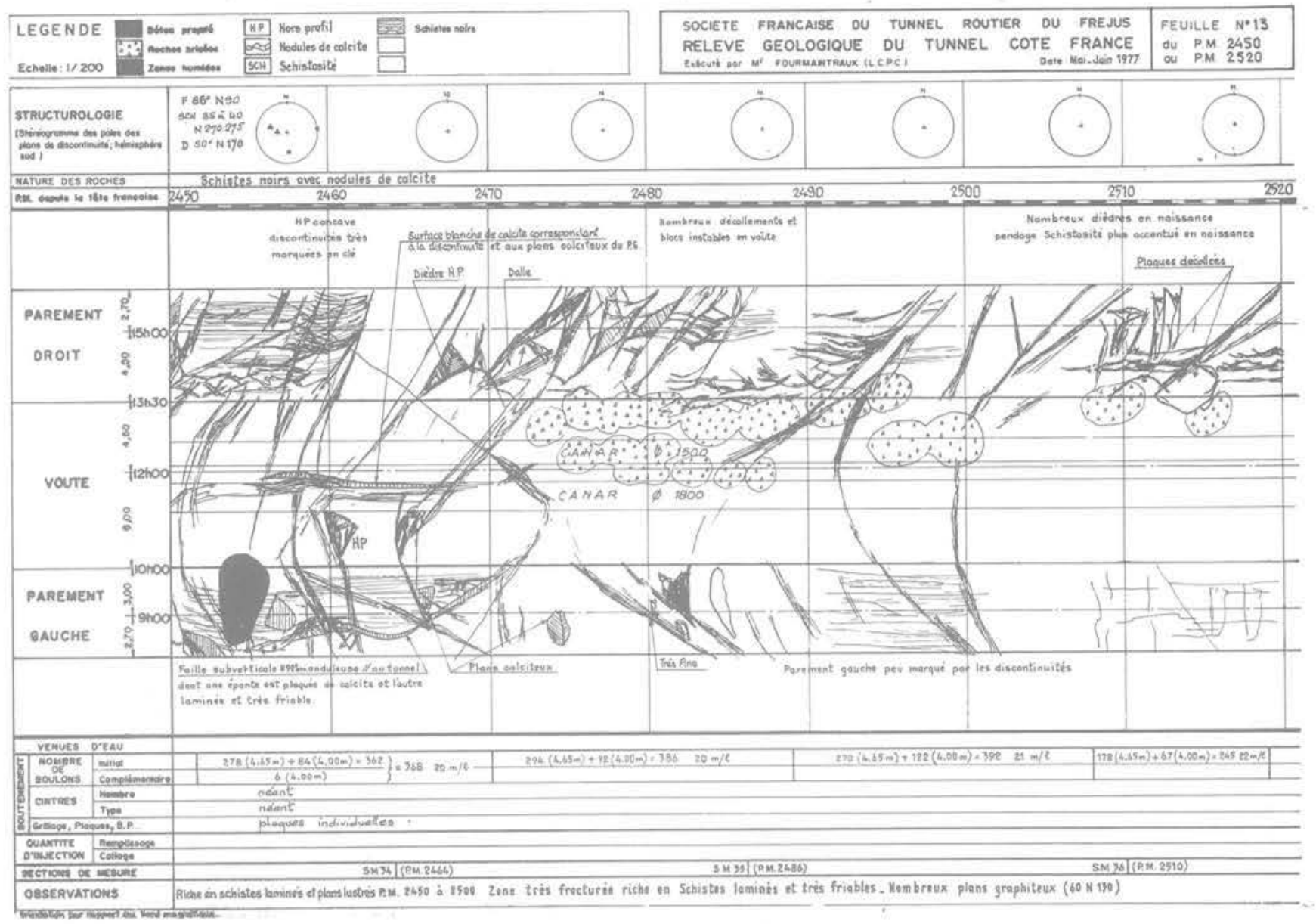

Fig. 12

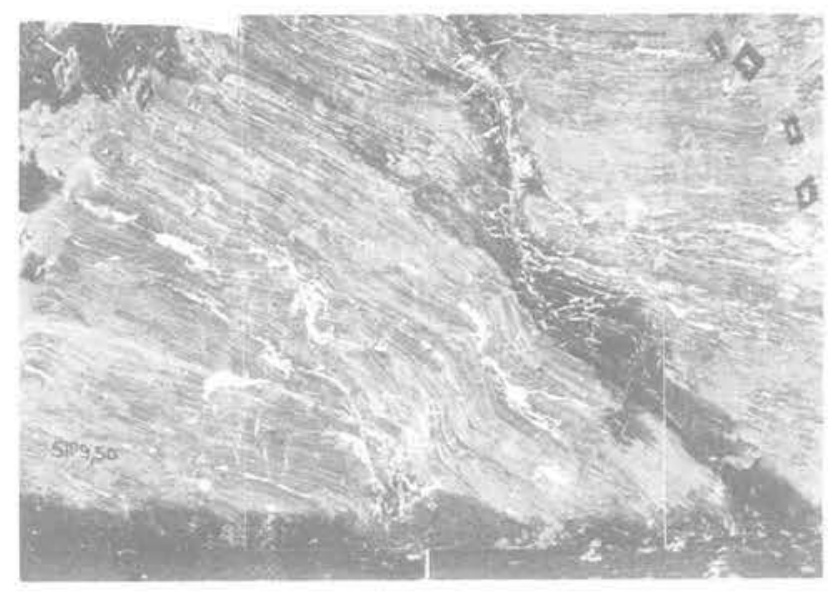

$\longmapsto$

Fig. 13 Détail du front au PM 5109,5

\subsubsection{Composition minéralogique}

Provenant du métamorphisme léger de formations marneuses et marnocalcaires plus ou moins sableuses, ces schistes contiennent essentiellement des minéraux phylliteux (20 à $50 \%$ de micas du type muscovite et chlorite), des carbonates (20 à $80 \%$ de calcite) accompagnés en plus faible quantité de quartz (5 à $30 \%$ ) et de produits charbonneux (anthracite, graphite) $(<5 \%)$ et de pyrite.

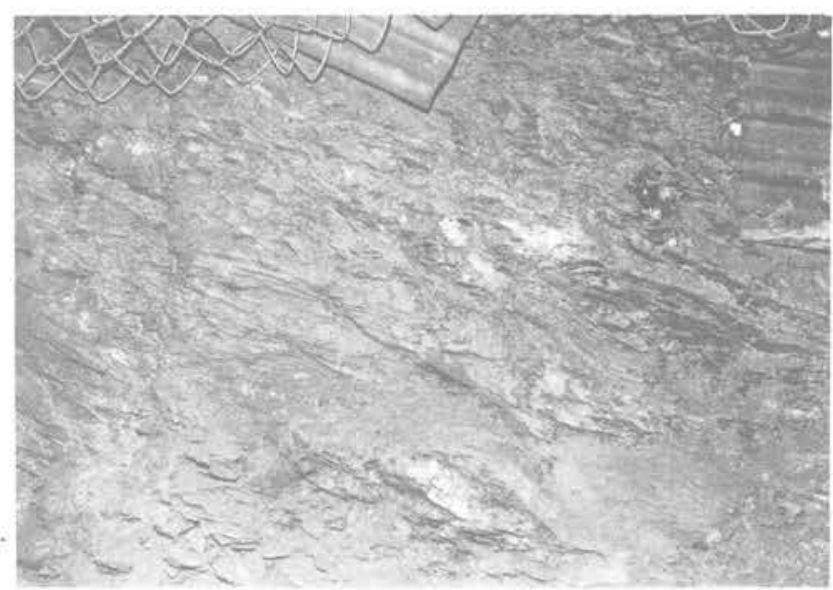

Fig. 14 Faciès phylliteux des schistes lustrés. Aspect du parement au PM 5087

\begin{tabular}{l|r}
\hline \multicolumn{1}{c|}{ Minéraux } & \multicolumn{1}{c}{ Teneur } \\
\hline Minéraux phylliteux & \\
(muscovite, chlorite) & 20 à $50 \%$ \\
Calcite & 20 à $80 \%$ \\
Quartz & 5 à $30 \%$ \\
Produits charbonneux & $5 \%$ \\
Pyrite & $1 \%$ \\
Feldspath (Albite) & $1 \%$ \\
Épidote & $1 \%$ \\
\hline
\end{tabular}


Un enrichissement local en pyrite associé à une disposition en lits fins riches en graphite et continus selon la structure feuilletée de la roche est vraisemblablement à l'origine des fortes valeurs de conductivité électrique de la roche en certaines zones (PM 5180 à 5350) qui ont entraîné des difficultés pour la mise à feu électrique des tirs. Enfin, on y trouve quelques minéraux accessoires typiques du métamorphisme : feldspath du type albite, et épidote.

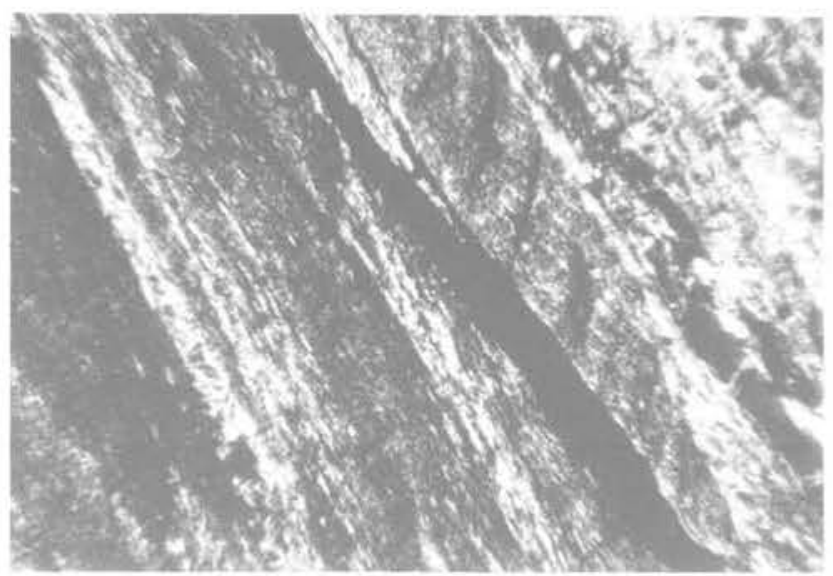

Fig. 15 Faciès phylliteux des schistes lustrés (échantilIon 169 - PM 5092,50). Lame mince en lumière polarisée. Grossissement x 75.

La schistosité est très marquée, les lits de mica, soulignés de noir par les produits charbonneux, sont disposés en fuseaux torsadés parallèles.

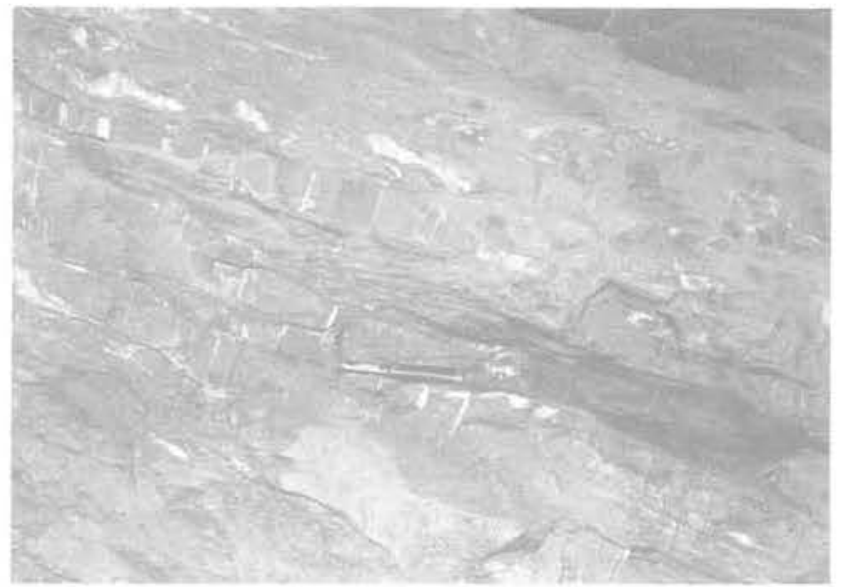

Fig. 16 Faciès «calcschiste» des schistes lustrés. Vue du front au PM 6500
Les variations des proportions relatives des deux constituants principaux, micas et calcite, correspondent à des termes soit plus micacés soit plus calcaires de la même série des schistes lustrés. Des variations dues aux déformations tectoniques du massif peuvent être également notées (fig. 14, 15, 15b, 16, 16b).

L'étude minéralogique en lame mince de quelques 250 échantillons prélevés au long de l'ouvrage a permis d'établir le diagramme de la figure 17.

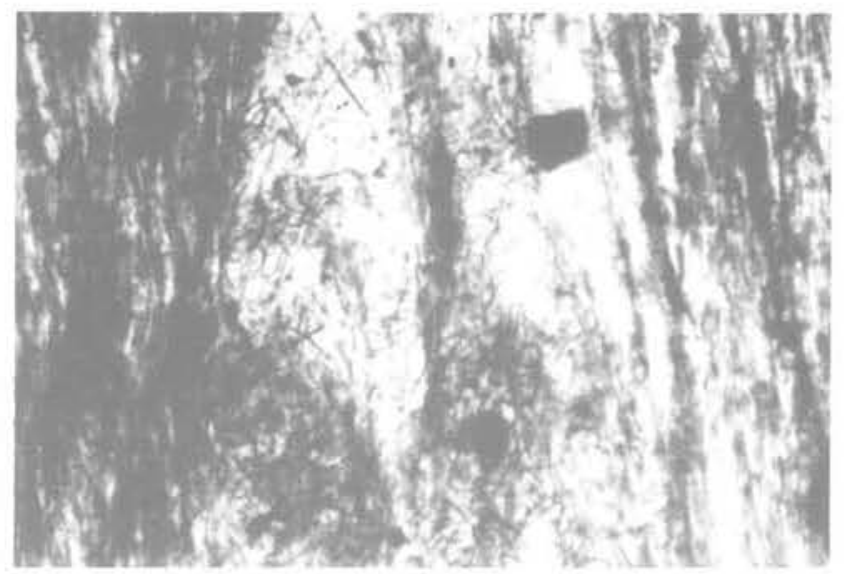

Fig. 15 bis Faciès phylliteux des schistes lustrés (échantillon 169 - PM 5092,50). Lame mince en lumière naturelle. Grossissement $\times 500$.

- Cristaux aciculaires de rutile inclus dans le quartz

- lits de mica avec matière charbonneuse

- dans le quartz se détachent des cristaux de pyrite noirs et trapus

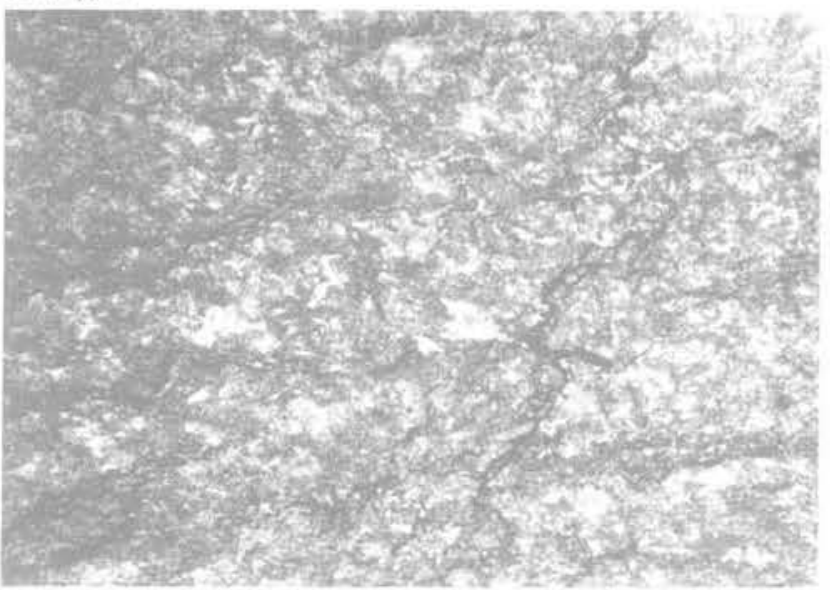

Fig. 16 bis Faciès calcaire des schistes lustres léchantilIon 213 - PM 5760). Lame mince en lumière naturelle. Grossissement x 25 .

Roche essentiellement constituée de carbonates; quelques cristaux de quartz (blancs) et des micas soulignés de matière charbonneuse noire et définissant une foliation fruste ; une foliation plus ancienne est encore décelable

Fig. 17 Variation de la composition minéralogique

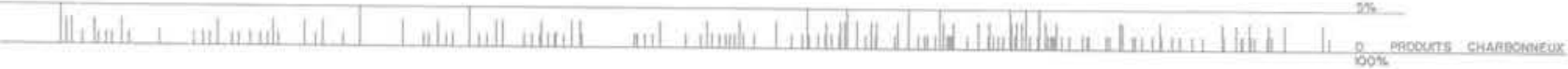

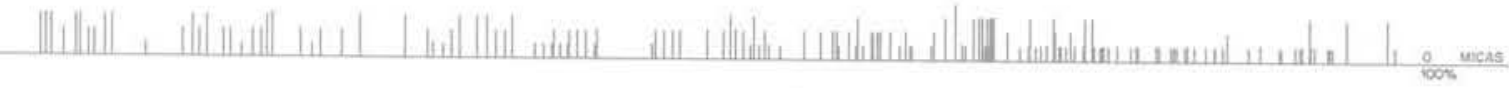

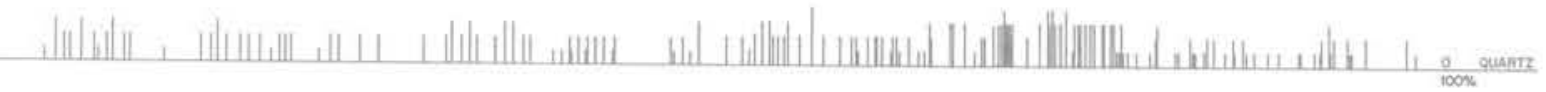

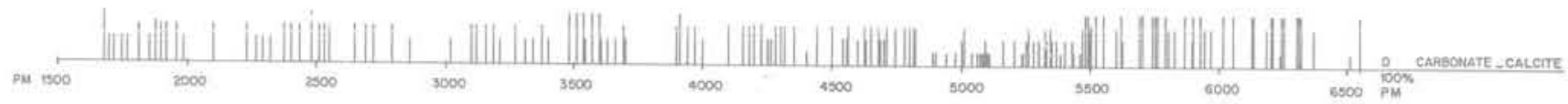




\subsection{Caractéristiques mécaniques des schistes lustrés}

L'influence de l'anisotropie de structure des schistes, sur leur comportement mécanique, est mise en évidence par les essais mécaniques: essais de compression simple et triaxiale, mesures de déformation au cours des essais de compression, mesure de la vitesse des ondes longitudinales, tant in situ qu'en laboratoire.

L'influence de la composition minéralogique semble également mise en évidence par les essais précédents; il est cependant nécessaire de procéder à des mesures complémentaires avant de pouvoir avancer des éléments chiffrés.

\subsubsection{Résistance à la rupture en compression simple}

Les échantillons testés proviennent de sondages carottés effectués aux points métriques 2100,2800 , $3300,4155,5500,5868,6000$, ainsi que de blocs de marinage prélevés avant le point métrique 1950 .

La résistance en compression simple a été mesurée sur des éprouvettes d'élancement égal à 2, l'effort étant appliqué soit parallèlement soit perpendiculairement au plan de schistosité.

Pour les deux orientations testées, on observe une dispersion importante des résultats puisque les résistances s'échelonnent entre 20 et $130 \mathrm{MPa}$ pour les éprouvettes sollicit ǰes perpendiculairement au plan de schistosité et entre 10 et $120 \mathrm{MPa}$ pour les éprouvettes sollicitées parallèlement à ce même plan. II semble difficile, compte tenu d'une telle dispersion, de faire apparaître une influence de l'anisotropie, puisque les plages de variation se recouvrent très largement. D'autre part la résistance la plus faible est obtenue lorsque la compression s'exerce obliquement par rapport au plan de schistosité.

En étudiant cependant les histogrammes de ces résistances (fig. 18 et 19), on fait apparaitre des différences significatives, qui mettent en évidence une tendance de la roche à être moins résistante lorsqu'elle est sollicitée parallèlement au plan de schistosité que Iorsqu'elle est sollicitée perpendiculairement à ce plan : cette tendance apparaît lorsque l'on considère les maxima des deux histogrammes; en effet, le maximum du premier histogramme (compression parallèle à la schistosité) est compris entre 30 et $50 \mathrm{MPa}$, alors que le maximum du second histogramme (compression perpendiculaire à la schistosité) est compris entre 50 et $80 \mathrm{MPa}$. Cette même tendance se confirme en éliminant les résultats extrêmes, on constate alors que pour la roche sollicitée parallèlement à la schistosité, $80 \%$ des résistances mesurées sont comprises entre 10 et $70 \mathrm{MPa}$, alors que pour la roche sollicitée perpendiculairement à la schistosité, $87 \%$ des résistances mesurées sont comprises entre 30 et $100 \mathrm{MPa}$.

\subsubsection{Déformabilité des schistes}

La déformabilité des schistes a été étudiée en mesurant, à l'aide d'extensomètres électriques, les déformations longitudinales et transversales des échantillons soumis aux essais de compression.

Si l'on exprime ces résultats en termes de Module de Young, on constate une dispersion importante pour les deux orientations testées :

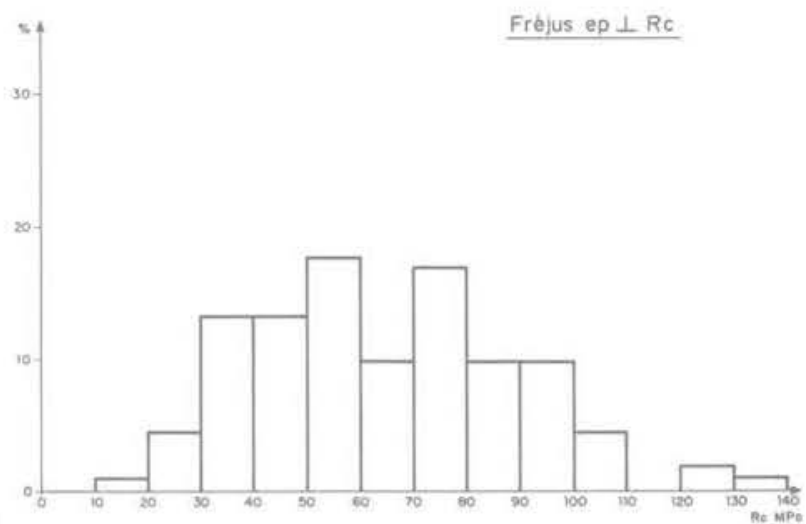

Fig. 18 Histogramme des résistances en compression simple (effort perpendiculaire à la schistosité)

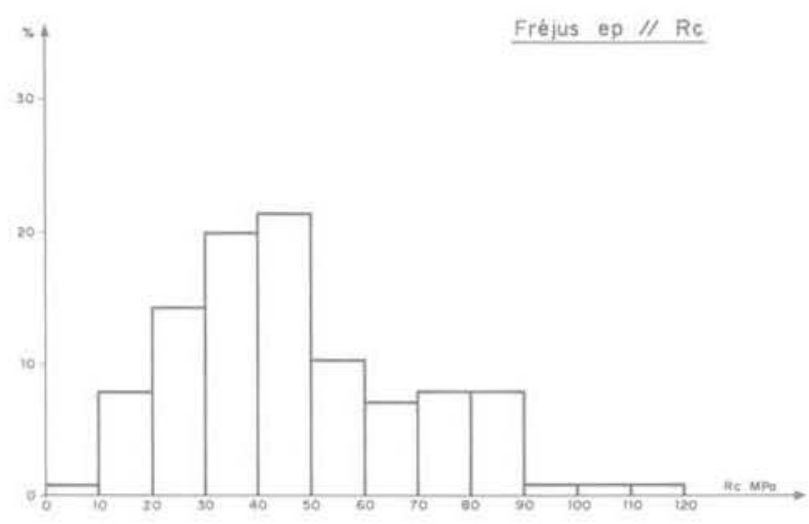

Fig. 19 Histogramme des résistances en compression simple (effort parallèle à la schistosité)

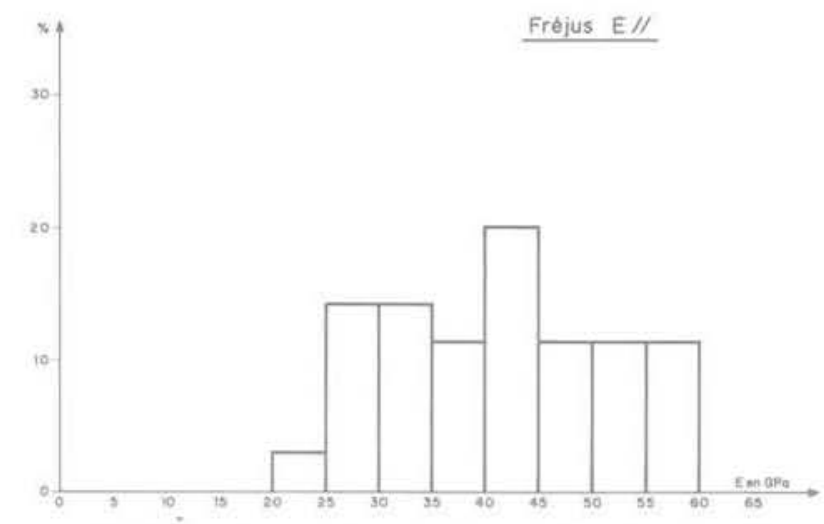

Fig. 20 Histogramme des modules de Young leffort parallèle à la schistosité)

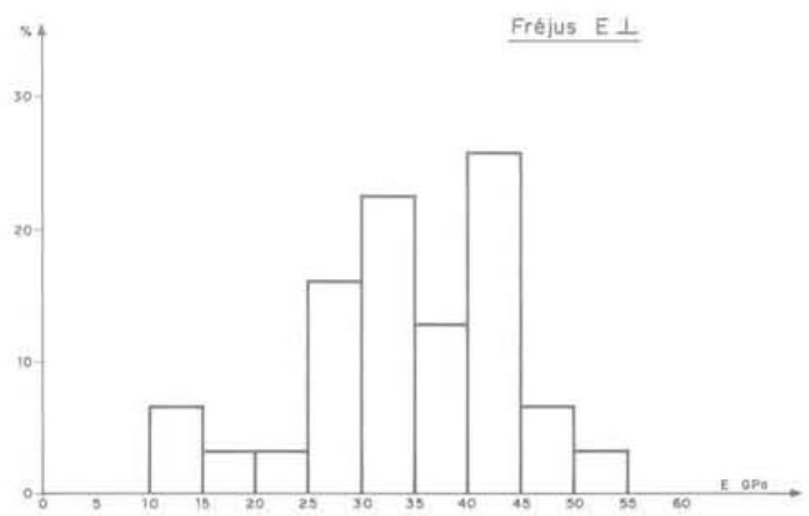

Fig. 21 Histogramme des modules de Young (effort perpendiculaire à la schistosité) 


$$
\begin{array}{r}
10000 \mathrm{MPa}<\mathrm{E} \perp<55000 \mathrm{MPa} \\
25000 \mathrm{MPa}<\mathrm{E} / /<60000 \mathrm{MPa} \text {, }
\end{array}
$$

et la zone de recouvrement de ces deux plages de variation, de 20000 à $55000 \mathrm{MPa}$ concerne environ $90 \%$ des échantillons de chacune des orientations (fig. 20 et 21).

En termes de Module de Young, l'influence de l'anisotropie ne semble donc se manifester que sur les valeurs extrêmes. En revanche, si l'on examine les courbes efforts-déformations, la différence de comportement devient évidente; on constate en effet l'existence d'une phase de serrage importante pour les éprouvettes sollicitées perpendiculairement au plan de schistosité alors que cette phase est inexistante pour les éprouvettes sollicitées parallèlement au plan de schistosité (fig. 22).

En fin de compte, c'est sur la déformation avant rupture que l'on observe une différence essentielle entre les échantillons sollicités perpendiculairement et parallèlement à la schistosité. En effet, la conjonction des trois paramètres que l'on vient d'évoquer : serrage, tendance à un module plus faible, tendance à une résistance plus grande aboutit au résultat qu'en moyenne la déformation avant rupture est trois fois plus importante pour la roche sollicitée perpendiculairement à la schistosité que pour la roche sollicitée parallèlement à la schistosité (fig. 22).

\subsubsection{Célérité des ondes longitudinales}

De nombreuses mesures ont été faites tant au L.C.P.C. qu'au Centre de Mécanique des Roches de I'E.N.S.M.P.; pour s'en tenir aux faits les plus saillants, il suffit d'observer que l'on constate une nette différence entre la célérité des ondes longitudinales suivant qu'elles se propagent parallèlement ou perpendiculairement à la schistosité :

Dans le premier cas : $4000 \mathrm{~m} / \mathrm{s} \leqslant \mathrm{V}_{\mathrm{L}} \leqslant 6000 \mathrm{~m} / \mathrm{s}$.

Dans le second cas : $1300 \mathrm{~m} / \mathrm{s} \leqslant V_{\mathrm{L}} \leqslant 3000 \mathrm{~m} / \mathrm{s}$.

Cette différence n'est pas surprenante et peut être mise en parallèle avec la différence entre les modules tangents à l'origine des courbes efforts-déformations pour les deux orientations testées (fig. 22).

\subsubsection{Comportement du schiste au voisinage de l'excavation}

L'axe longitudinal du tunnel étant à peu près parallèle au plan de schistosité, le profil d'excavation est donc tangent à ce plan, en un point dont la position varie de $12 \mathrm{~h} 30$ à $14 \mathrm{~h}$ suivant l'inclinaison $\left(15^{\circ}\right.$ à $\left.40^{\circ}\right)$ de ce plan sur l'horizontale $(12 \mathrm{~h}=$ clé de voûte; $15 \mathrm{~h}=$ intersection du profil avec le rayon horizontal). Le comportement mécanique du schiste permet de comprendre qu'une instabilité et des phénomènes de rupture se manifestent dans cette zone de 12 h 30 à $14 \mathrm{~h}$ où les contraintes tangentielles sont parallèles au plan de schistosité, direction de moindre résistance.

L'ordre de grandeur des contraintes tangentielles, en considérant un recouvrement de $1000 \mathrm{~m}$ et un facteur de concentration de 2, à la paroi de l'excavation, est voisin de $60 \mathrm{MPa}$ et, par conséquent, suffisamment élevé pour provoquer des ruptures dans la zone considérée.

L'idée vient donc tout naturellement, pour améliorer la stabilité, de solidariser les bancs entre eux et d'exercer un confinement, à l'aide de boulons; on pense d'emblée aux boulons à ancrage ponctuel qui sont les

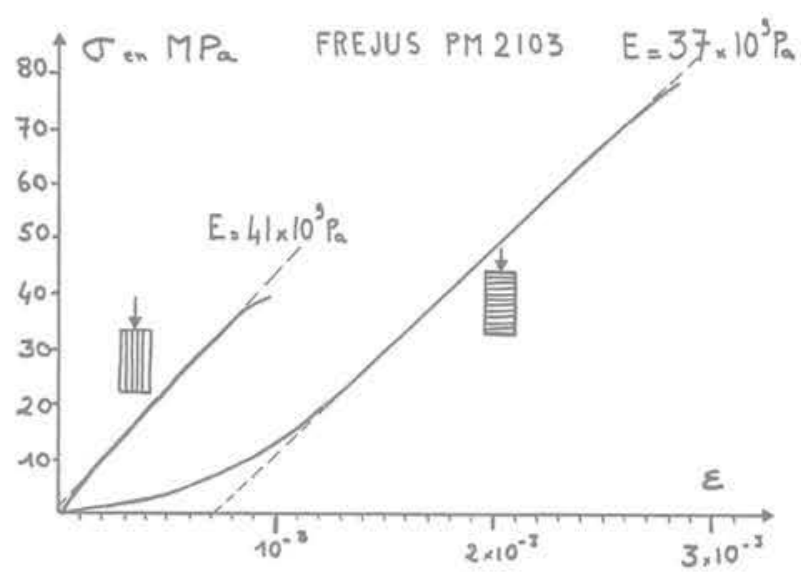

Fig. 22 PM 2103 - Courbes efforts-déformations en compression simple

plus rapides à poser, dans la mesure où le schiste est assez résistant pour encaisser les contraintes locales élevées qui règnent au voisinage d'un ancrage.

\section{Aperçu des méthodes d'exécution du tunnel}

\subsection{Excavation et soutènement provisoire}

L'excavation est faite à l'explosif, en pleine section, avec un bouchon à trous parallèles et des couronnes d'abattage successives, suivant la méthode du découpage soigné. Le plan de tir (fig. 23) comporte 160 trous environ, pour une volée de $4,50 \mathrm{~m}$. Les trous $\varnothing 48 \mathrm{~mm}$ et le trou central $\varnothing 152 \mathrm{~mm}$ sont forés par deux pantofores électro-hydrauliques Montabert équipés chacun de cinq bras et d'une nacelle. Le chargement des cartouches préfabriquées en gaines plastiques se fait à partir de deux plates-formes auto-élévatrices C.F.I.A.

Après le tir, l'aspiration des fumées et l'arrosage du tas, on procède au marinage à l'aide d'une chargeuse électrique Broyt X4 (godet de 2,5 $\mathrm{m}^{3}$ ) qui charge des dumpers CAT 613 de 20 t de charge utile (fig. 24).

Deux des douze dumpers déposent leur charge en cordon le long d'un piédroit (fig. 25) à $100 \mathrm{~m}$ du front et retournent aussitôt au front de façon que la chargeuse ne soit jamais arrêtée (méthode de laminage). Les autres évacuent leur chargement hors du tunnel puis évacuent ensuite le cordon pendant les phases suivantes du cycle.

La purge de la calotte et du front se fait à l'aide d'une purgeuse mécanique Pingon dont le bras a été mis spécialement au point pour ce chantier. II en résulte un gain important en efficacité par rapport à la méthode traditionnelle et surtout une plus grande sécurité pour le personnel qui n'est pas exposé pendant cette phase dangereuse. On procède ensuite à la mise en place du soutènement provisoire ( 20 boulons $\varnothing 20 \mathrm{~mm}$ de $4,65 \mathrm{~m}$ par mètre de tunnel, 15 plaques et $18 \mathrm{~m}$ de grillage $\varnothing 5 \mathrm{~mm}$ ) à l'aide de deux boulonneurs Montabert équipés chacun de trois bras et d'une nacelle (fig, 26).

Le cycle-type se décompose comme suit, pour 11 volées hebdomadaires de $4,5 \mathrm{~m}$ (pour 16 postes de travail) et un avancement mensuel de $200 \mathrm{~m}$. 


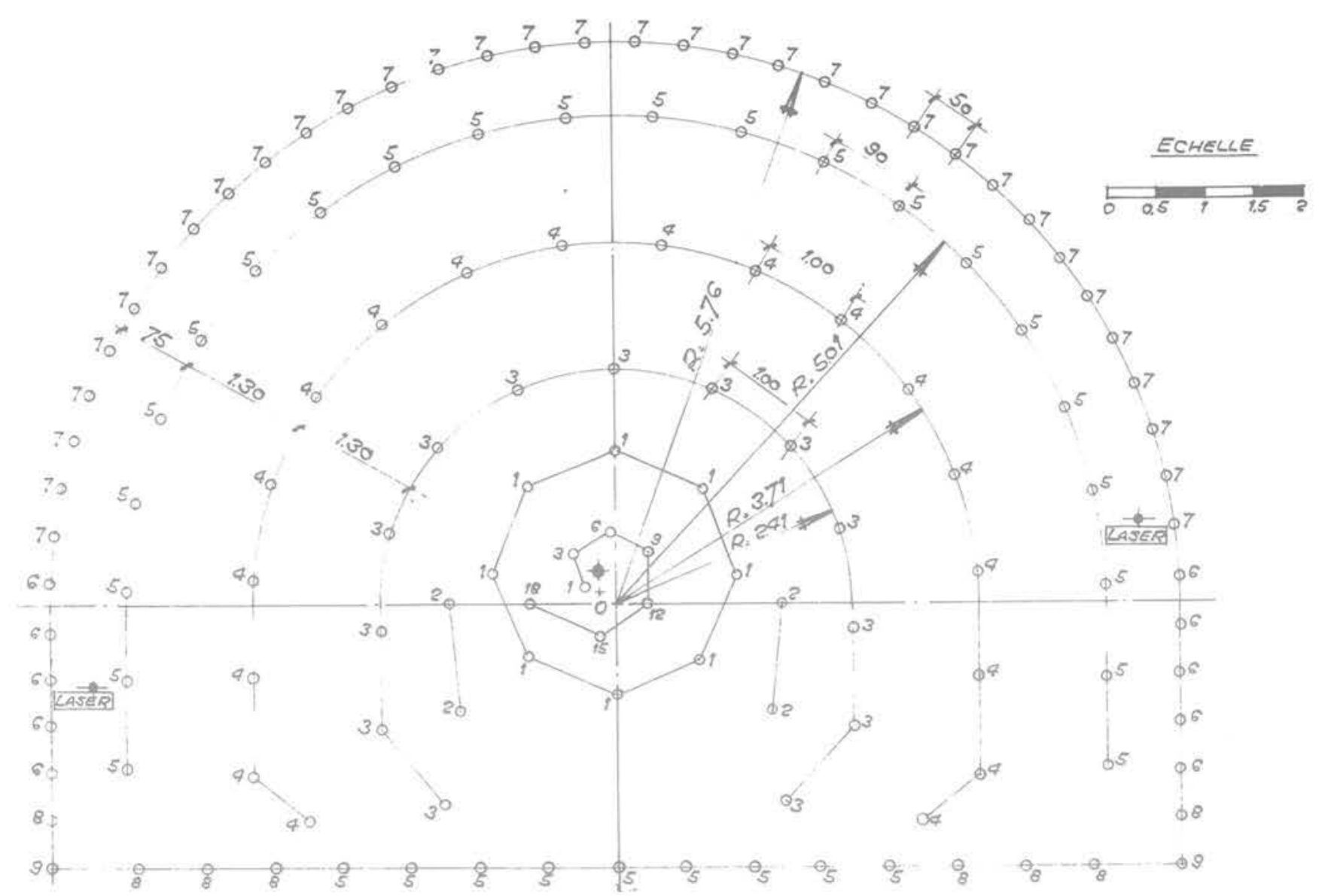

Fig. 23 Plan de tir

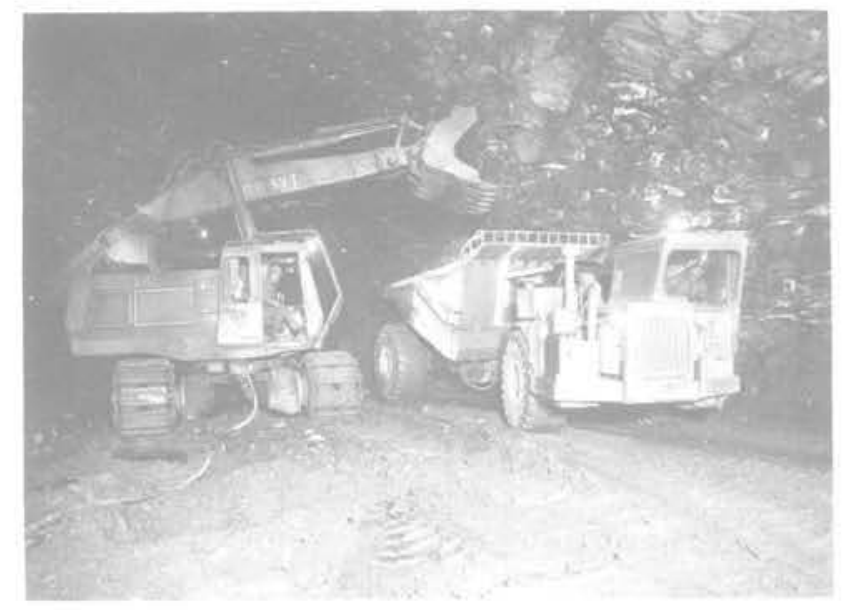

Fig. 24 Chargement des dumpers

Fig. 26 Boulonnage de la volite

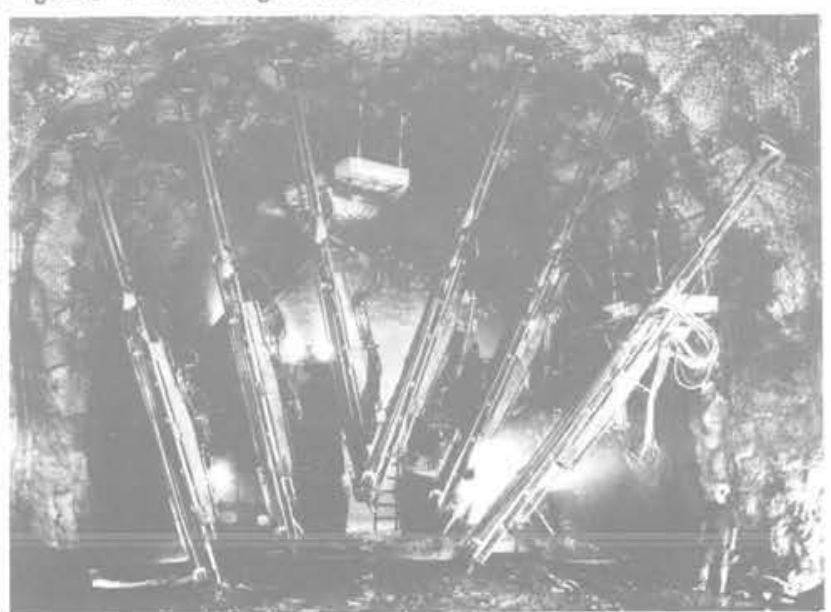

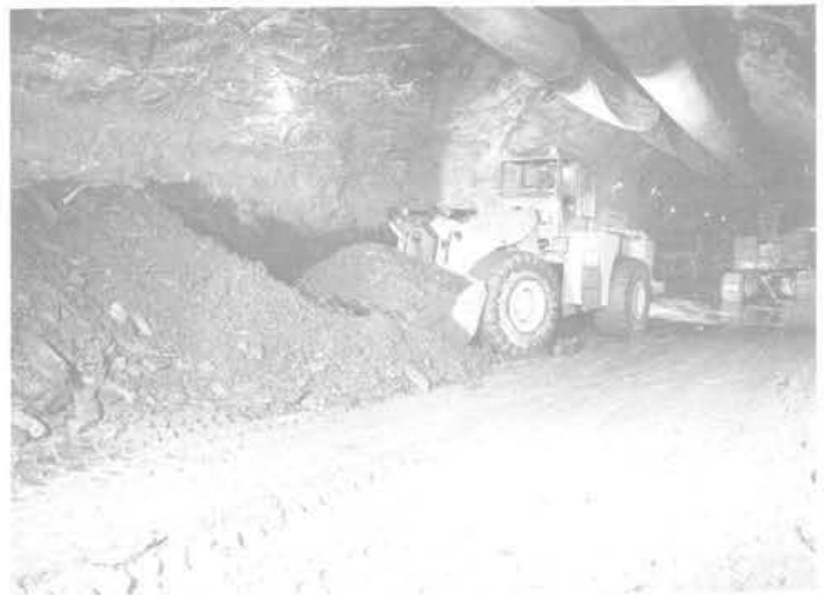

Fig. 25 Mise en cordon des déblais

Fig. 27 Coulée des banquettes de fondation de la voute et du béton de roulage

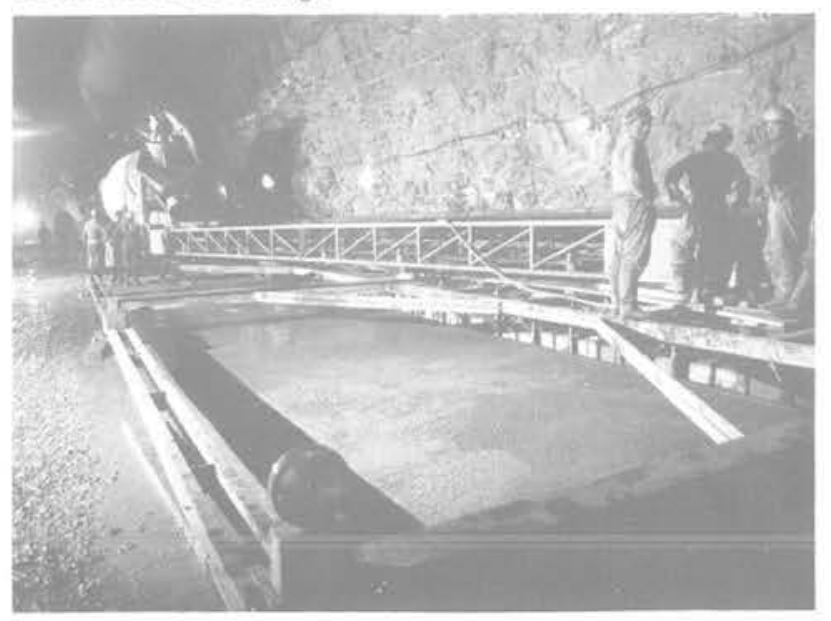




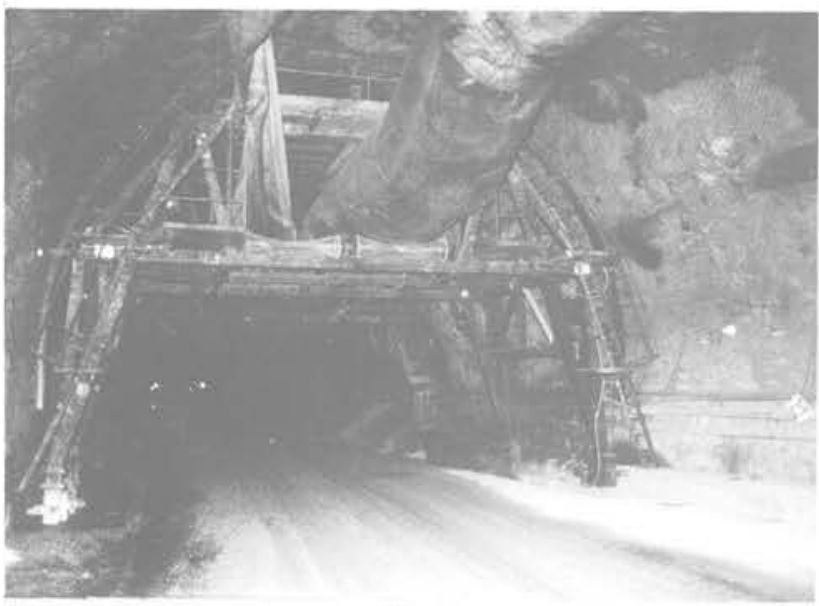

Fig. 28 Portique de reprofilage

Fig. 30 Bétonnage de la dalle de plafond

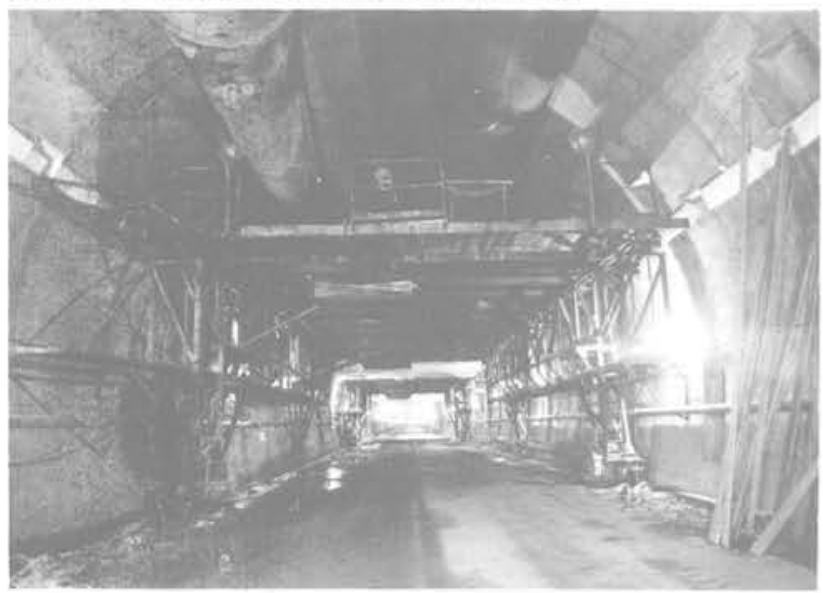

Mise en place des pantofores

Perforation de la volée $(4,5 \mathrm{~m})$

Retrait des pantofores

Chargement de la volée

2 h 30

Tir-Évacuation des fumées

$1 \mathrm{~h} 15$

0 h 35

Purge - Marinage

3 h 40

Mise en place des boulonneurs

Perforation-pose des boulons

et plaques

2 h 15

Pose du grillage

Retrait des boulonneurs

$1 \mathrm{~h}$

Total

11 h 15

\subsection{Revêtement}

Au-delà de la zone de stationnement et d'entretien des engins d'excavation, on rencontre les chantiers de bétonnage qui se succèdent dans l'ordre suivant:

\subsubsection{Banquettes de fondation de la voûte et béton de roulage}

Exécution par demi-largeur de façon à laisser toujours libre une voie de circulation et une chicane pour passer d'un côté à l'autre (fig. 27).

\subsubsection{Reprofilage}

C'est-à-dire mise au gabarit de l'excavation avant bétonnage de la voûte, avec repiquage des sous-profils à l'aide de deux portiques roulant sur les banquettes en béton (fig. 28).

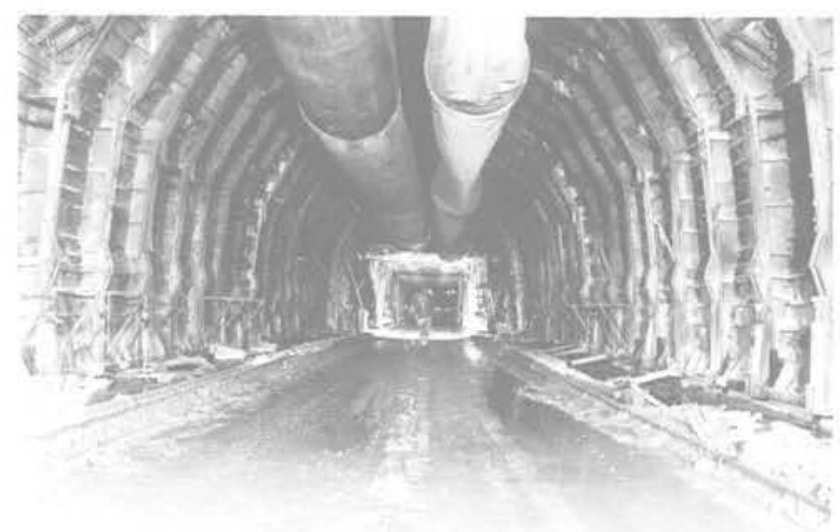

Fig. 29 Bétonnage de la voute

Fig. 31 Bétonnage de la cloison entre les conduits d'air frais et d'air vicie

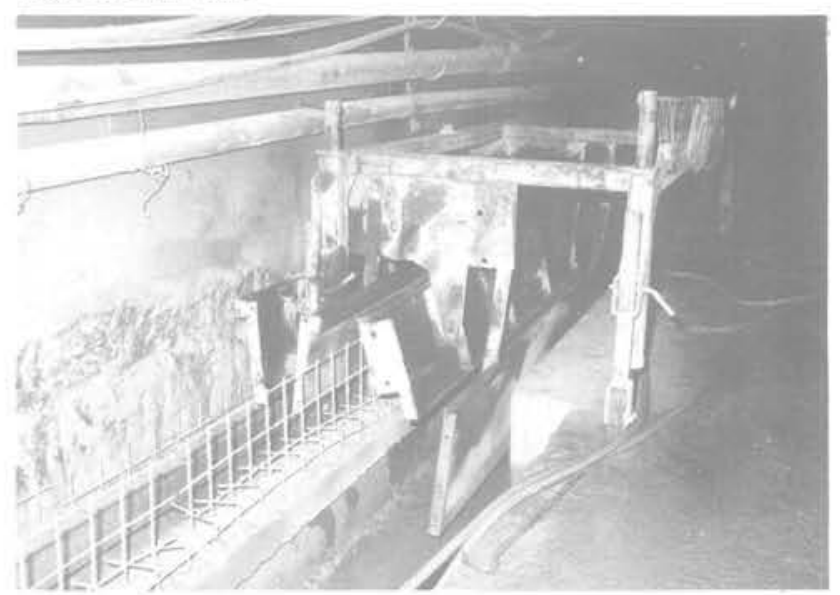

\subsubsection{Bétonnage à la pompe de la voûte}

Par anneaux de $13,50 \mathrm{~m}$ de longueur à l'aide de trois cintres télescopiques Blaw-Knox (fig. 29).

\subsubsection{Bétonnage à la pompe de la dalle du plafond}

Par éléments de $18 \mathrm{~m}$ (fig. 30 ).

\subsubsection{Bétonnage à la pompe de la cloison et exécution des joints d'étanchéité en Sikaflex}

Plus loin, on trouve les chantiers d'exécution des trottoirs et des caniveaux de drainage (fig. 31).

\subsection{Chaussée}

Plus tard, on viendra rajouter aux $40 \mathrm{~cm}$ moyens du béton de roulage, $21 \mathrm{~cm}$ de chaussée souple.

\subsection{Organisation des ateliers successifs}

La figure 32 montre en élévation et en plan les divers ateliers rencontrés, à partir du front, vers la sortie du tunnel. L'opération en cours, au front, est le marinage après le tir et la ventilation d'aspiration des fumées. A 50 mètres du front, se trouve le portique de ventilation qui porte le ventilateur d'air vicié, les extrémités des ventubes d'air frais et d'air vicié et divers appareils électriques. Une bonne soixantaine de mètres est ensuite réservée au stockage des déblais en laissant une voie libre pour la circulation. On trouve ensuite, sur $150 \mathrm{~m}$, une zone de stockage et d'entretien des 


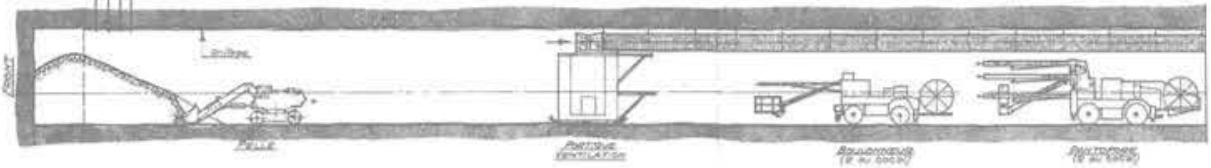

We en par

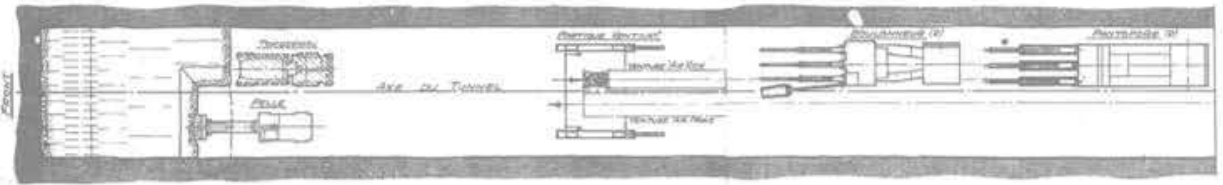

Fig. 32a Abattage - marinage - soutènement

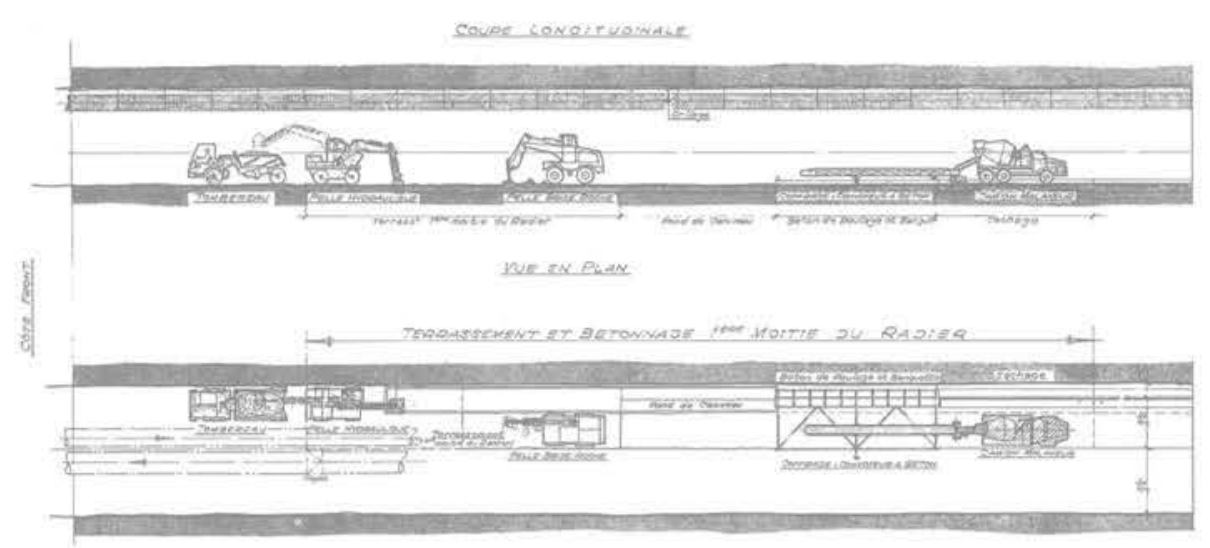

Fig. 32b Béton de roulage et banquettes (1ère moitié)

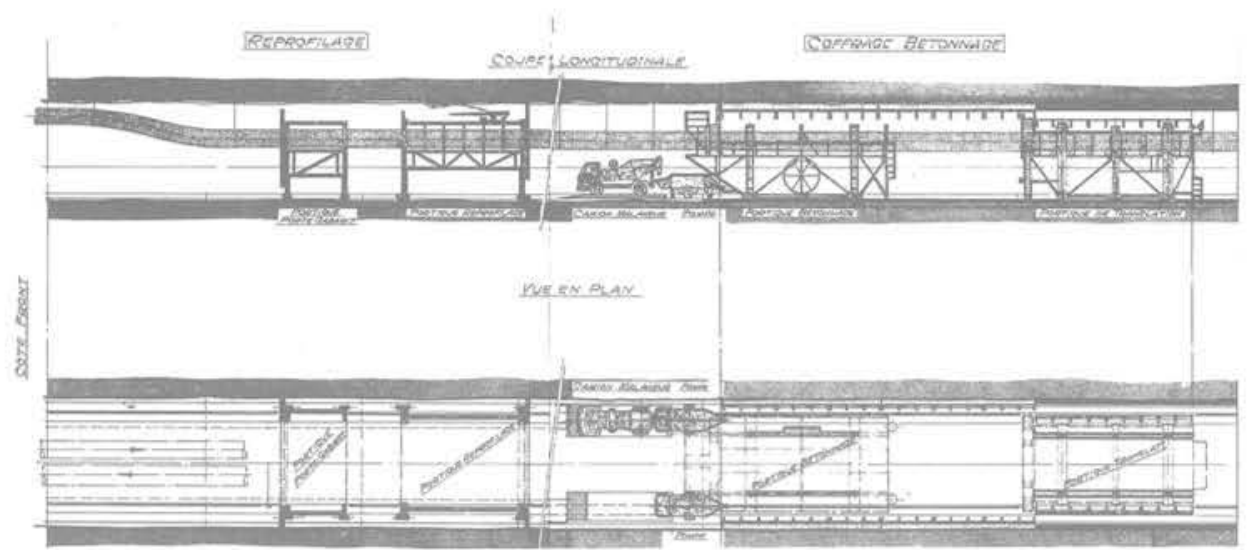

Fig. 32c Revêtement vou te et piédroits

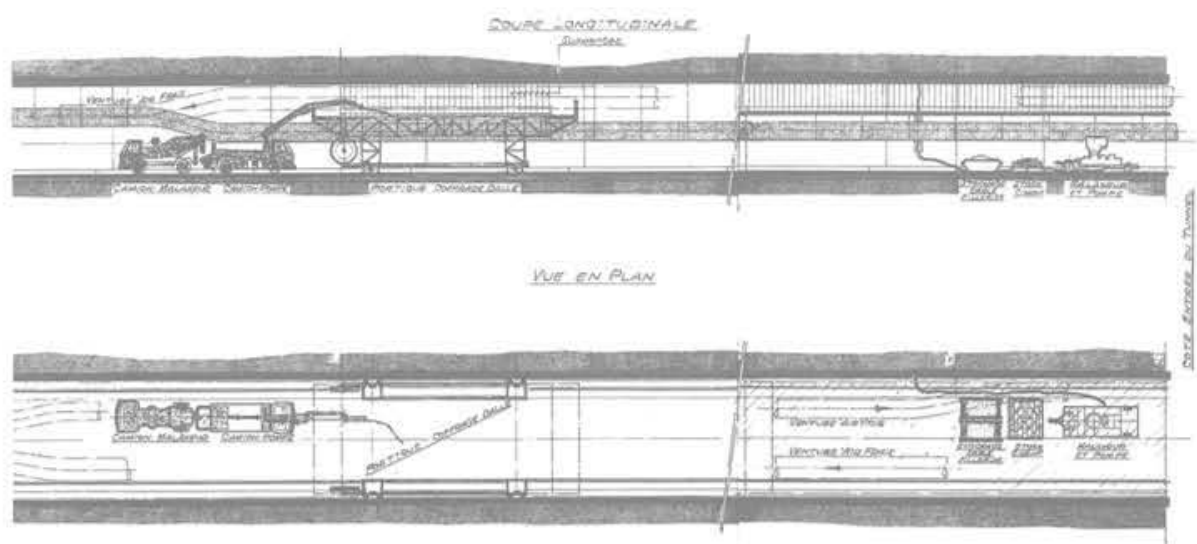

Fig. 32d Dalle pour conduits ventilation et injections 
engins du front (à l'exception des dumpers dont l'entretien est fait à l'extérieur du tunnel).

A $270 \mathrm{~m}$ du front, commence le chantier de bétonnage du béton de roulage et des fondations de la voûte. $\mathrm{Ce}$ chantier de 216 mètres de long comporte pour chaque demi-largeur successive, une zone d'excavation complémentaire et de nettoyage, une zone de bétonnage et une chicane de circulation permettant aux engins de passer d'une voie à l'autre.

A 500 mètres du front, on trouve le chantier de reprofilage avec un portique de détection qui précède un portique de travail; ce dernier est muni à l'arrière d'un profilé ayant la forme de l'excavation minimale souhaitée et ne peut avancer tant que le reprofilage n'est pas exécuté.

A 600 mètres du front environ, on trouve le chantier de bétonnage de la voûte avec ses 3 coffrages et ses 2 portiques de manutention et de soutien.

Vers 700 mètres du front, commence le chantier de bétonnage de la dalle du plafond.

Vers 900 mètres, on trouve le chantier d'injection pour remplissage des vides qui subsisteraient entre la voûte et le rocher.

Entre $950 \mathrm{~m}$ et $1250 \mathrm{~m}$ du front, sont exécutés la cloison du plafond, les ragréages, les joints d'étanchéité en Sikaflex.

Vers $1300 \mathrm{~m}$ ou $1400 \mathrm{~m}$, on trouve les ventilateurs relais qui reçoivent l'air frais par le conduit définitif terminé et le renvoient vers le front dans des ventubes. La longueur des ventubes ne peut guère dépasser $1400 \mathrm{~m}$ sans risquer de voir le débit d'air chuter dangereusement.

\section{Les mesures géotechniques}

\subsection{Introduction}

De nombreuses mesures géotechniques ont été effectuées tout au long du chantier, sur l'excavation non revêtue. Ces mesures in situ ont eu pour objet de mieux connaître et de suivre le comportement de l'excavation, de déterminer puis d'adapter le soutènement provisoire et enfin de surveiller l'excavation non revêtue pour assurer la sécurité du chantier jusqu'à la mise en place du revêtement. Elles ont connu un caractère systématique, à partir du moment où le tunnel a pénétré dans les schistes lustrés à forte couverture (PM 1710).

Enfin, des bases de mesures ont été mises en place dans les zones revêtues pour permettre de surveiller le comportement de l'ouvrage pendant le chantier puis après sa mise en service.

\subsection{Les mesures de convergence sur excavation non revêtue}

\subsubsection{Technologie}

Des déformations du profil de l'excavation se sont essentiellement produites sur les côtés ouest dans le secteur $13 \mathrm{~h}-15 \mathrm{~h}$ au sein de la voûte et à la naissance du parement, où le plan de schistosité est tangent au profil. Les bancs de schistes sont comprimés par les efforts tangentiels qui s'exercent parallèlement au plan de schistosité : il se décollent ainsi les uns des autres

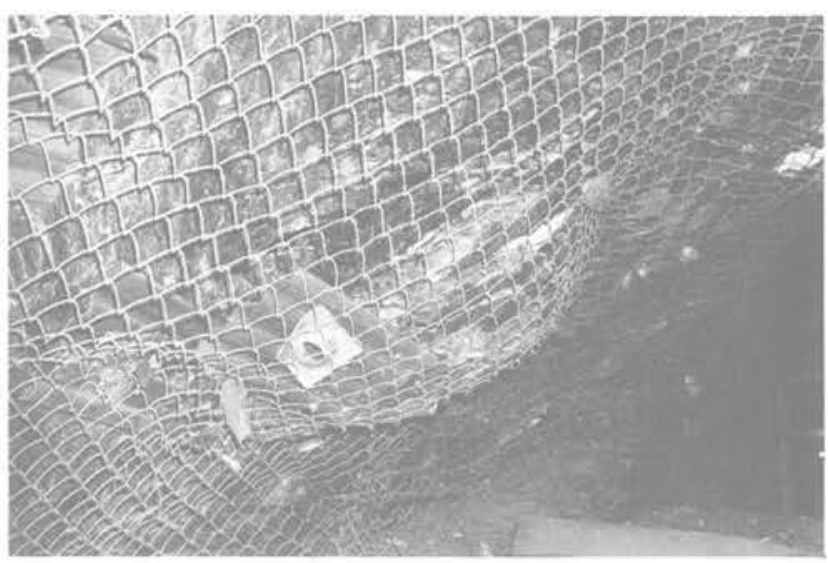

Fig. 33 Désordres dans le secteur de la naissance droite de la voûte

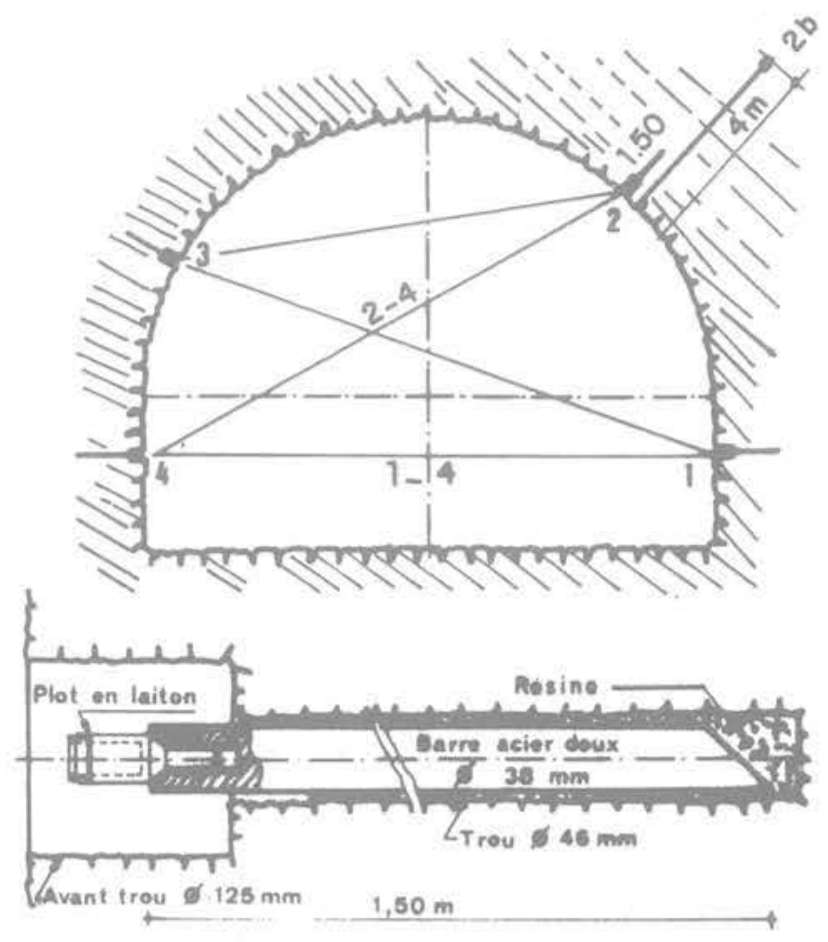

Fig. 34 Plots de mesures de convergence

et se rompent alors par flambage (fig. 11 en haut et photo fig. 33). Cette particularité liée à la position de la galerie par rapport à la structure du massif a déterminé, en partie, la disposition des plots de mesure de convergence du profil destiné à suivre le comportement global de l'excavation (fig. 34). Chaque section de mesure comporte quatre plots ( $n^{\circ} 1$ à 4 ) respectivement situés à $15 \mathrm{~h}, 13 \mathrm{~h} 30,10 \mathrm{~h}$ et $9 \mathrm{~h}$ dans le profil. Ces plots sont constitués d'une barre d'acier doux de diamètre $\varnothing 38 \mathrm{~mm}$ et de $1,50 \mathrm{~m}$ de long; scellée à la résine sur toute sa longueur, cette barre est équipée d'une tête filetée en laiton qui permet d'y fixer les extrémités du distancemètre à fils LPC type D01 (fig. 35). La mesure initiale est réalisée au plus près du front de taille (entre 2 et $5 \mathrm{~m}$ ), les sections de mesures sont régulièrement implantées tous les 30 mètres.

Entre les PM 1750 et 6500,40000 mesures furent réalisées sur près de 150 sections, permettant de suivre les convergences depuis l'excavation jusqu'à la mise en place du revêtement définitif, c'est-à-dire en moyenne pendant une durée de 3 à 4 mois. 


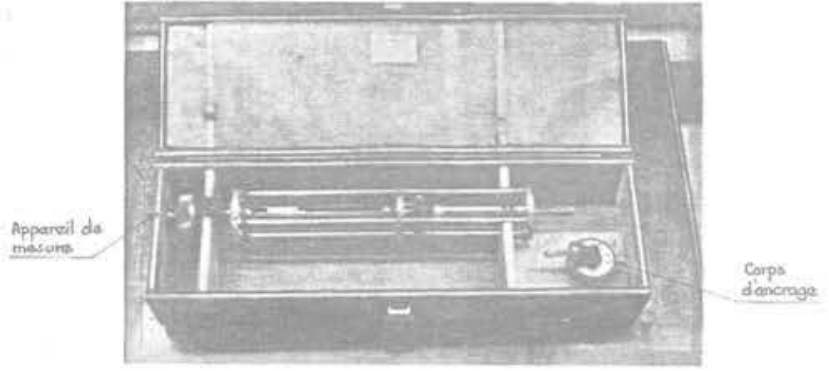

Photo 1 Distancemètre D01

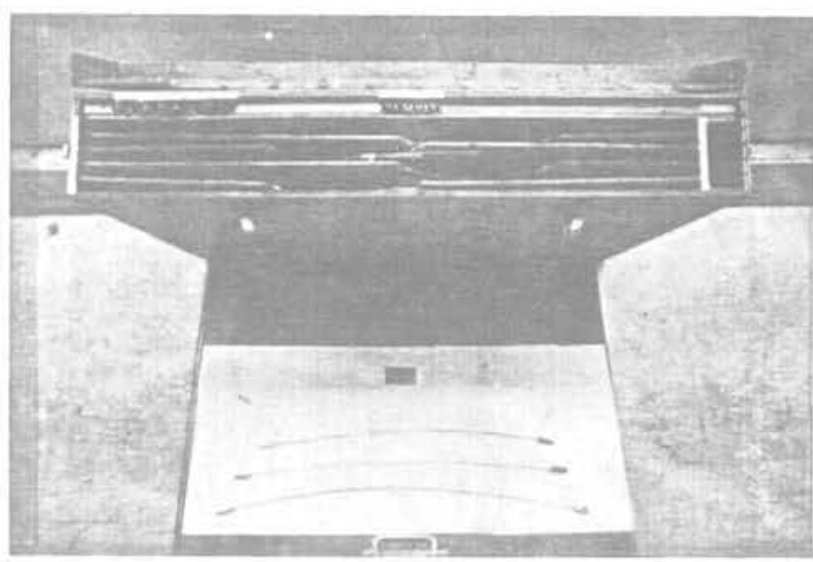

Photo 2 Jeux de fils et de rallonges etalonnes

Fig. 35 Lot de mesure du distancemètre D01

La réalisation pratique de telles mesures implique une coordination minutieuse des interventions entre l'Entreprise et le Maître d'CEuvre qui y consacra une équipe de quatre personnes utilisant trois véhicules dont une nacelle auto-élévatrice et les appareils de mesure mis à disposition par le C.E.T.E. de Lyon.

Ces mesures ont donné aux responsables du chantier des informations constantes sur les conditions de stabilité de l'excavation.

\subsubsection{Résultats}

Les mesures ainsi réalisées permettent l'établissement pour chaque section, des courbes de convergence en fonction du temps (fig. 36). La convergence $\mathrm{C}$ a été maximale sur la base $(2-4)$ où elle a atteint une valeur de $50 \mathrm{~cm}$, soit $4,5 \%$ de l'ouverture $(D=12 \mathrm{~m}) \mathrm{de}$ l'excavation, la majorité des valeurs de convergence ne dépassant pas $25 \mathrm{~cm}$.

Entre les piédroits, sur la base (1-4), les convergences furent de plus faible ampleur avec une valeur maximale de $35 \mathrm{~cm}$ et la majorité des valeurs inférieures à $10 \mathrm{~cm}$. Des mesures topographiques sur les plots permettent de constater que le plot 4 bouge peu et que la plus grande part de la convergence provient des mouvements du plot 2 .

\begin{tabular}{c|c|c}
\hline Base & \multicolumn{2}{|c}{ Convergence C } \\
\hline & Valeur maximale & $\begin{array}{c}\text { Majorité des } \\
\text { valeurs }\end{array}$ \\
$(2-4)$ & $50 \mathrm{~cm}$ & $<25 \mathrm{~cm}$ \\
$(1-4)$ & $35 \mathrm{~cm}$ & $<10 \mathrm{~cm}$ \\
\hline
\end{tabular}

La vitesse de convergence $\frac{\Delta \mathrm{C}}{\Delta \mathrm{T}}$ entre deux mesures successives a été calculée systématiquement; en effet, les courbes donnant les variations de la vitesse de convergence en fonction du temps présentent également un grand intérêt pour le suivi du creusement : un accroissement de la vitesse de convergence constitue une alerte qu'il convient d'interpréter. Ainsi sur la figure 37 le pic situé au $73^{\circ}$ jour correspond à une accélération passagère de la convergence provoquée par des opérations de reprofilage réalisées au droit de la section.

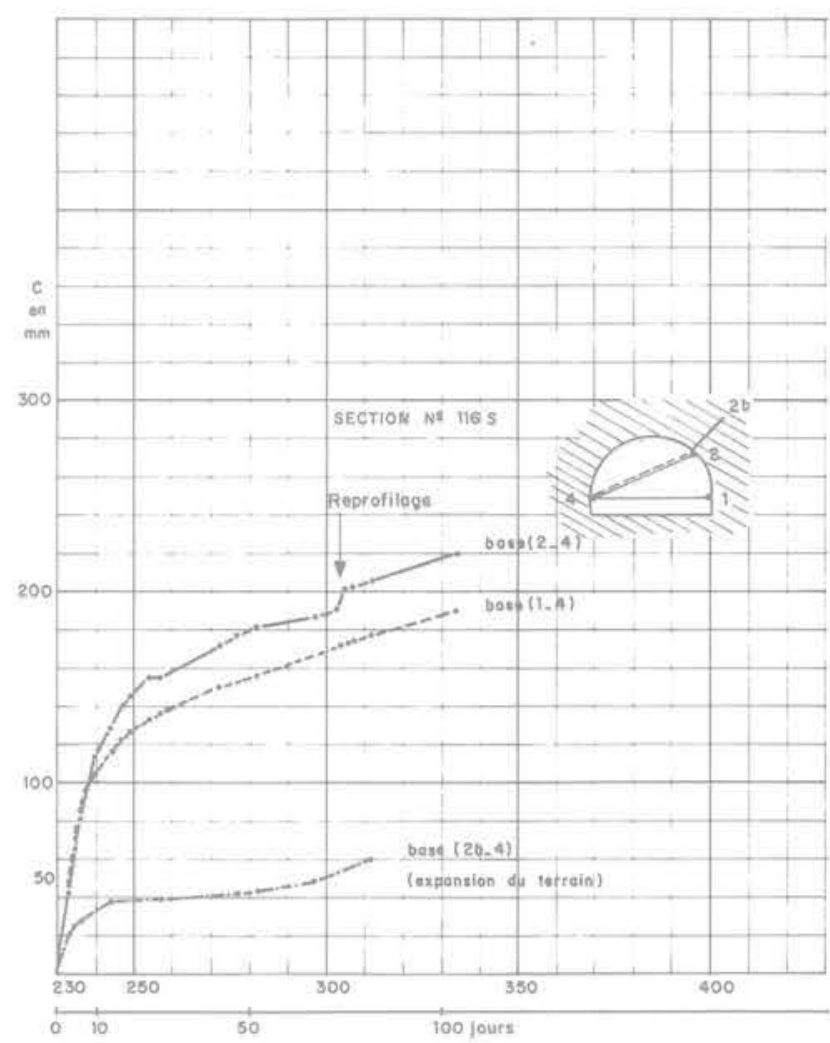

Fig. 36 Evolution des convergences en fonction du temps

Fig. 37 Evolution de la vitesse de convergence

VITESSE DE CONVERGENCE $\frac{\Delta C}{\Delta t}$

$$
\underset{\mathrm{mm} / \mathrm{I}}{\mathrm{EN}}
$$

20

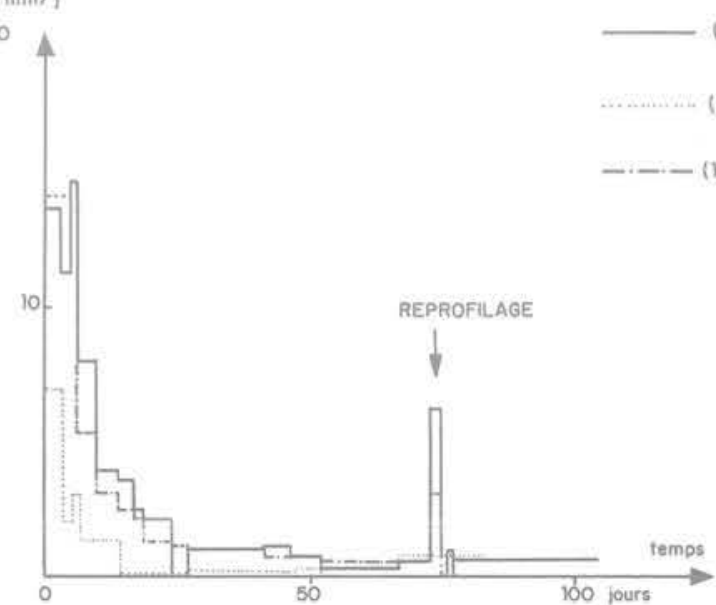



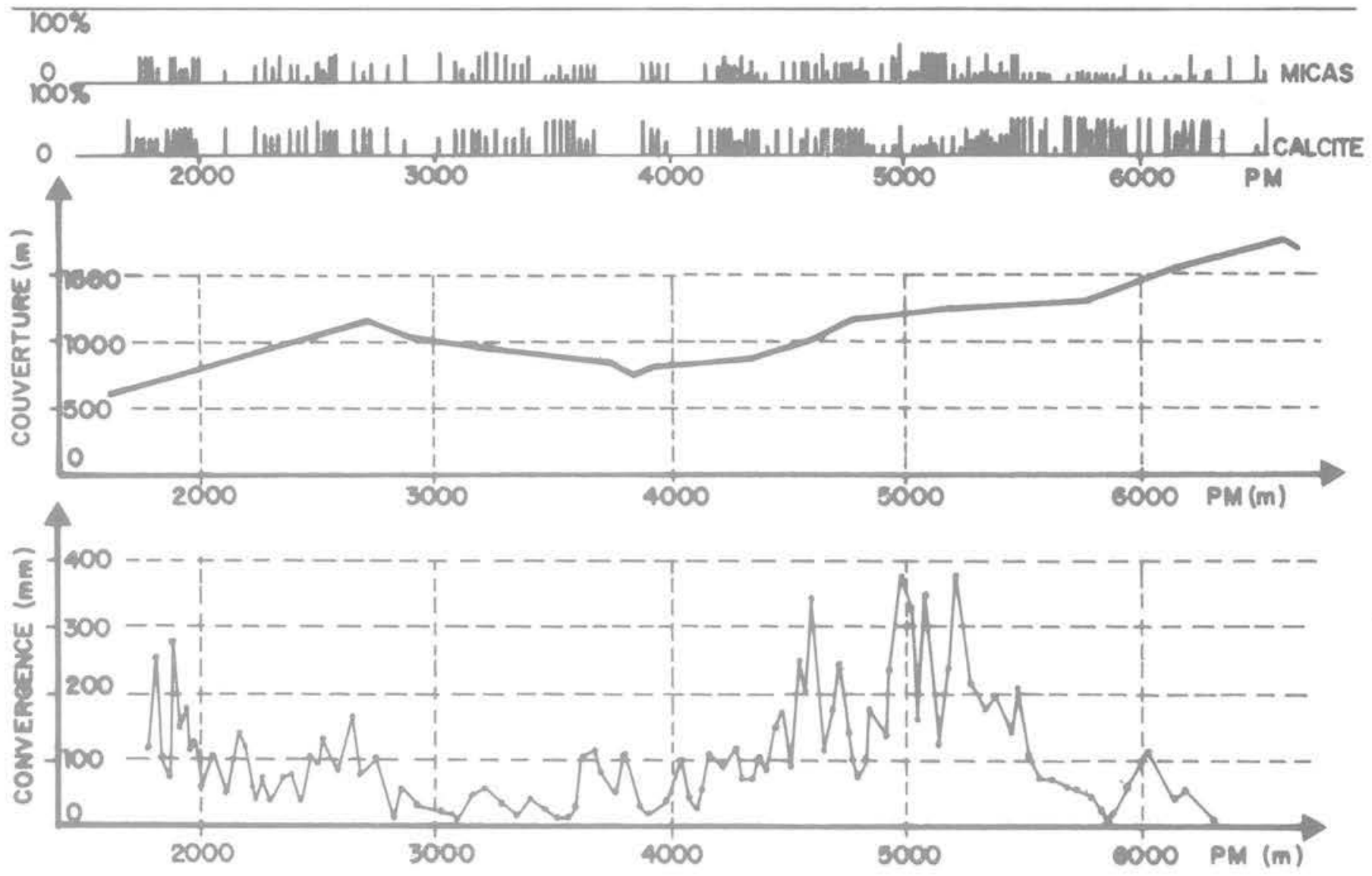

Fig. 38 Convergence à un mois

Fig. 39 Correspondance entre convergence, célérité sismique et composition minéralogique

\subsubsection{Convergence de la galerie et recouvre- ment}

Si on reporte les valeurs des convergences obtenues sur la base $(2-4)$ un mois après l'ouverture des sections, sur le profil en long de la galerie (fig. 38), il apparaît clairement qu'il n'y a pas de relation directe entre l'amplitude de la convergence en un point du tunnel et la hauteur du recouvrement au droit de ce point; on remarquera en particulier les valeurs faibles obtenues sur le dernier kilomètre où la couverture est la plus forte.

\subsubsection{Convergence et qualité des terrains}

Pour quantifier la qualité des terrains traversés, on a mesuré la célérité des ondes sismiques à l'aide d'un dispositif de sismique-réfraction. Ces mesures furent exécutées à la paroi de la galerie à travers le revêtement de béton définitif. Les dispositifs faisaient $30 \mathrm{~m}$ de long et les impacts étaient espacés de $5 \mathrm{~m}$. De cette façon, il fut possible de mesurer la célérité du massif intact en dehors de la zone perturbée par l'excavation. Sur une section de l'ouvrage de $1000 \mathrm{~m}$ de long avec de forts contrastes de convergence (entre les PM 4500 et 5500 ), on constate (fig. 39 ) que les zones à forte convergence correspondent à des zones où les célérités minimales ont été obtenues; une célérité inférieure à $4900-5000 \mathrm{~m} / \mathrm{s}$ semble être représentative de zones où la convergence au cours des cinq premiers jours fut supérieure à $50 \mathrm{~mm}$.

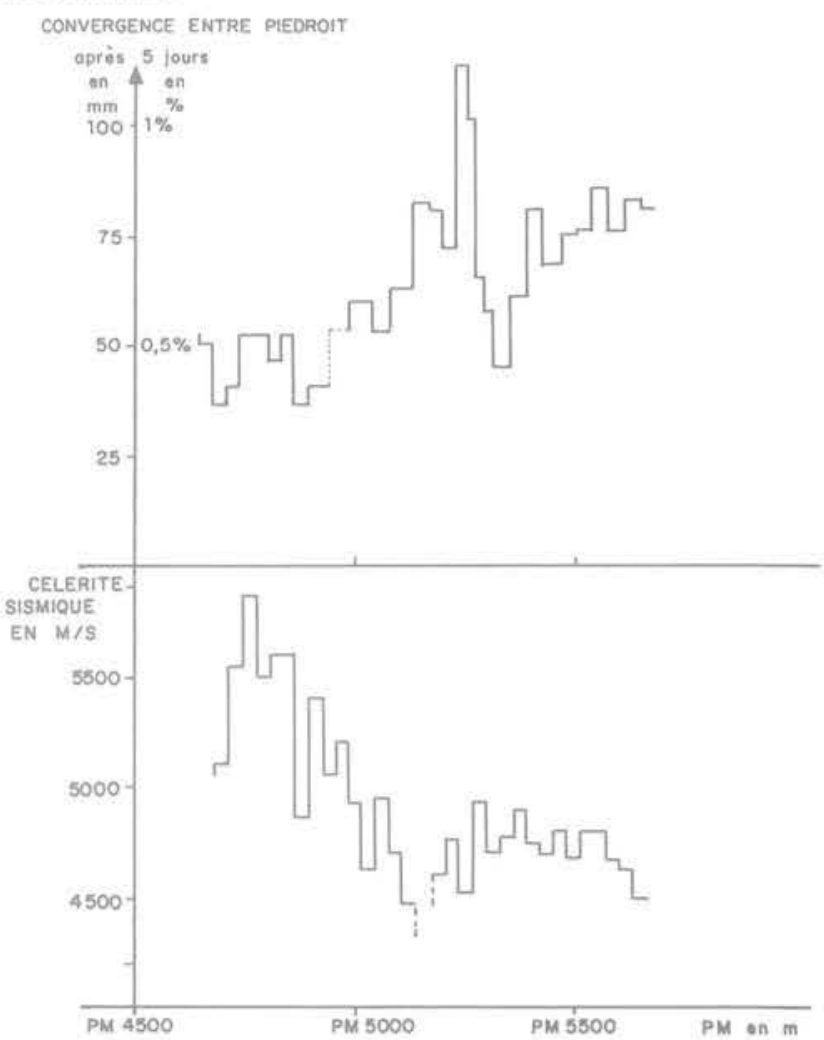

$100 \%$

MICAS

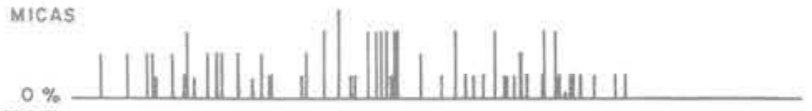

$100 \%$

CALCITE

$0 \%$

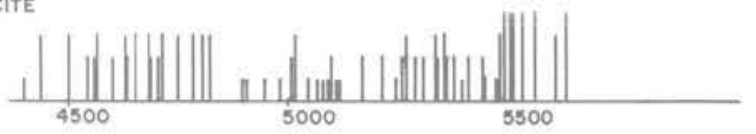




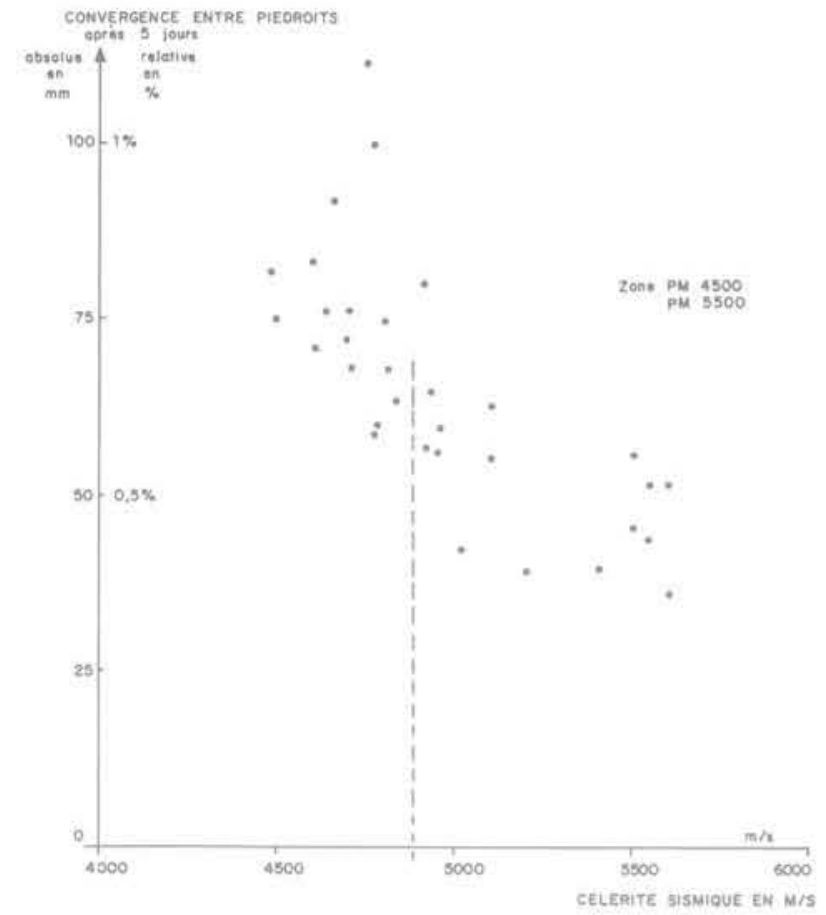

Fig. 40 Corrélation entre convergence et célérité sismique

II faut noter également que les zones à forte convergence sont le plus souvent situées dans des faciès phylliteux plus ou moins laminés.

Les déformations que traduisent ces convergences procèdent essentiellement de deux causes :

- l'une est due à l'exécution et à la progression des travaux d'excavation; elle est observable dans la zone d'influence du front de taille:

- l'autre correspond au comportement rhéologique du massif rocheux.

\subsubsection{Influence de la proximité du front de taille}

La progression du creusement provoque une modification des conditions d'équilibre du massif qui se manifeste par des mouvements de convergence en arrière du front. La distance à partir de laquelle la présence du front de taille n'influence plus de façon significative les déformations de convergence, a pu être étudiée à l'aide des mesures exécutées pendant les périodes comportant un arrêt et une reprise des travaux d'excavation. Par exemple, un arrêt de chantier de 22 jours a eu lieu au PM 5103,5: les courbes de convergence des trois sections les plus proches, SM 119, SM 118 et SM 116 situées respectivement à $6 \mathrm{~m}, 29 \mathrm{~m}$ et $63 \mathrm{~m}$ du front sont reportées sur la figure 41 .

La reprise de l'avancement s'accompagne d'un changement de courbure très nettement perceptible pour les deux premières sections les plus proches; la troisième située à $63 \mathrm{~m}$ ne réagit pas de façon sensible. Si on considère maintenant (fig. 42), les variations de la vitesse de convergence, l'effet de reprise est mieux marqué pour les sections 119 et 118; pour la section 116, la plus lointaine, une légère perturbation est décelable.

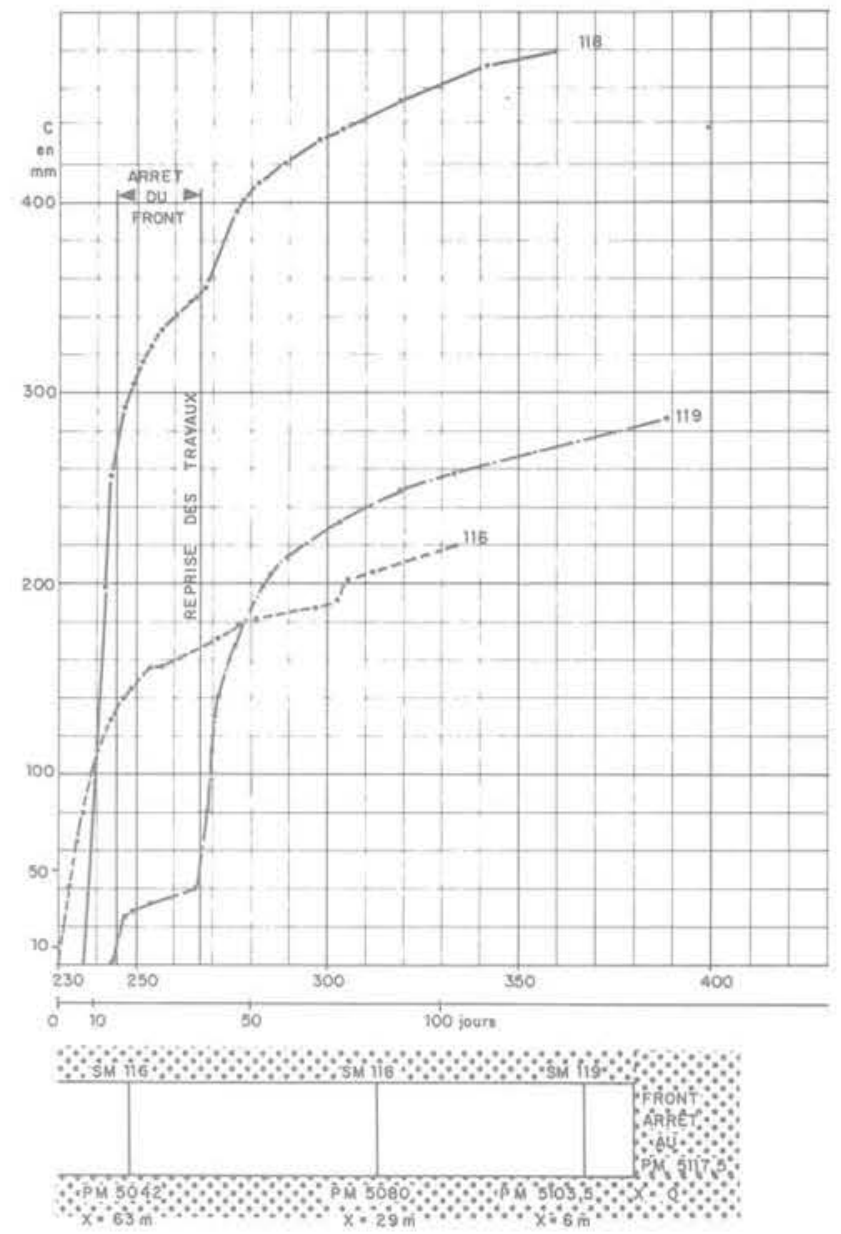

Fig. 41 Influence du front de taille - base 2 - 4

Au tableau II, sont reportées les valeurs de la vitesse de convergence aux cours des derniers jours d'inactivité et au cours des premiers jours de reprise.

Pratiquement, la distance d'influence du front de taille a été trouvée en moyenne de l'ordre de 50 à $70 \mathrm{~m}$ soit un peu plus de 5 fois l'ouverture de l'excavation. Cette distance est supérieure à la distance d'influence du calcul élastique : il est possible que les ébranlements provoqués par les tirs dans la roche et les boulons ancrés ponctuellement jouent un rôle pour accroître cette distance d'influence.

\begin{tabular}{cc|c|c}
\hline \multirow{2}{*}{$\begin{array}{c}\text { Section de Distance au } \\
\text { mesure } n^{\circ} \text { front }(\mathrm{m})\end{array}$} & \multicolumn{2}{|c}{ Vitesse de convergence $\frac{\mathrm{dC}}{\mathrm{dT}}$} \\
\cline { 3 - 4 } & $\begin{array}{c}\text { 年ant } \\
\text { la reprise des travaux } \\
(\mathrm{mm} / \mathrm{j})\end{array}$ \\
\hline SM 119 & 6 & 0,25 & $\begin{array}{c}\text { après } \\
\text { SM 118 }\end{array}$ \\
SM 116 & 63 & 1,00 & 5,12 \\
\hline
\end{tabular}

Tableau /I

\subsubsection{Comportement rhéologique du massif rocheux}

Durant ces arrêts de l'avancement et plus généralement en dehors de la zone d'influence du front de taille, l'évolution des convergences ne dépend plus 


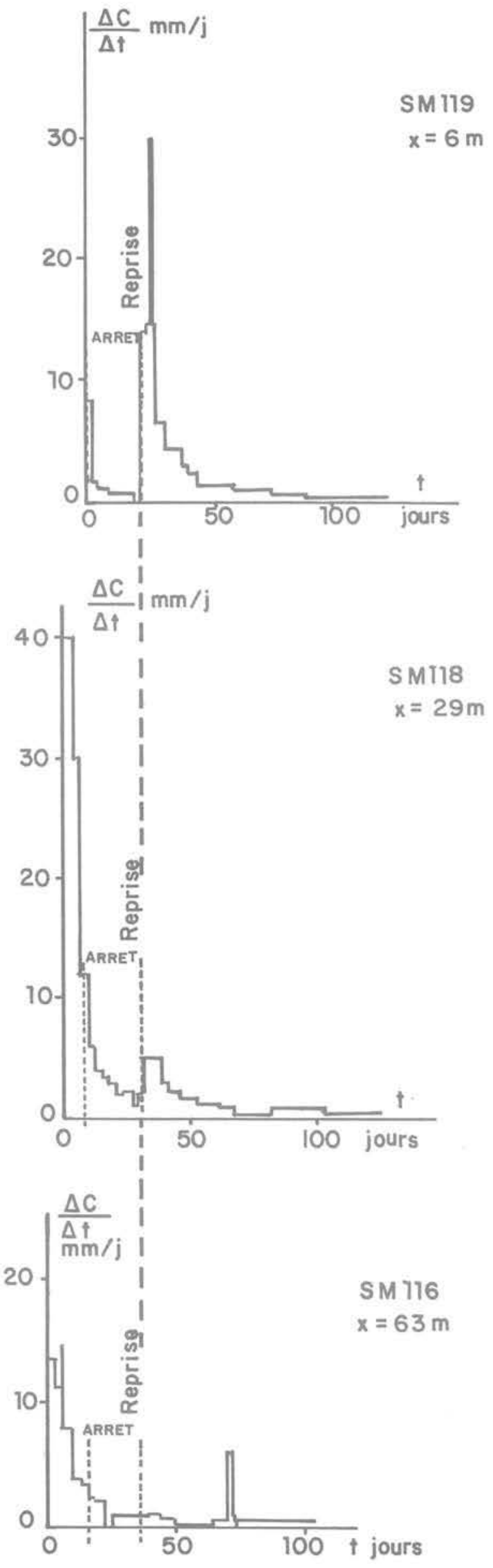

Fig. 42 Influence de la proximité du front sur la vitesse de convergence

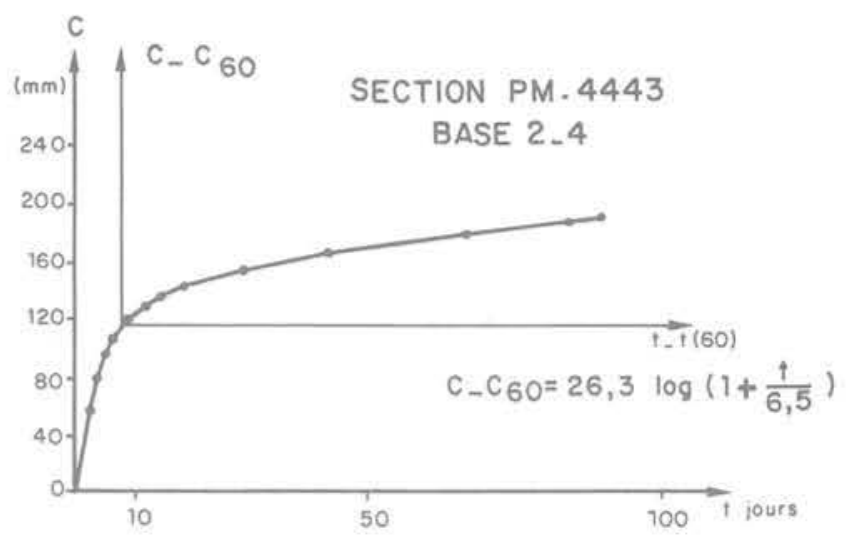

Fig. 43 Evolution de la convergence. Ajustement d'une loi logarithmique

que du comportement rhéologique du massif; elle correspond aux déformations différées résultant de l'évolution des terrains autour de l'excavation.

Pour représenter simplement cette évolution de la convergence $\mathrm{C}$ en fonction du temps $\mathrm{t}$, on a recherché une loi analytique; on constate ainsi que cette évolution a lieu à vitesse régulièrement décroissante et, compte tenu de la durée des mesures avant la mise en place du revêtement, il n'est pas possible de mettre en évidence de limite asymptotique. Un ajustement expérimental remarquablement précis (à quelques $1 / 10 \mathrm{~mm}$ près au maximum) avec une loi logarithmique est obtenu dans la plupart des cas, il est de la forme:

$$
\mathrm{C}-\mathrm{C}_{(60)}=\mathrm{B} \log \frac{\mathrm{t}}{\mathrm{T}}
$$

où $\mathrm{C}$ représente la convergence au temps $t$, ou $C_{(60)}$ est la convergence mesurée au moment où le front s'est éloigné de $60 \mathrm{~m}$ de la section de mesure et $\mathrm{B}, \mathrm{T}$ sont des constantes.

La figure 43 donne l'exemple d'un de ces ajustements (SM 98; PM 444) qu'on n'a pu vérifier évidemment que pendant une durée limitée à 4 ou 5 mois en raison de la mise en place du revêtement définitif.

\subsubsection{Prévision de la courbe de convergence après les premières mesures}

Du point de vue de la surveillance et de l'adaptation du soutènement (allongement limite des boulons), il est également important de pouvoir prévoir la convergence finale peu de temps après les premières mesures. Là encore, on a pu constater que pour un avancement normal et régulier du front, la convergence obéissait à une loi du type $\mathrm{C}=\mathrm{A} \log \left(1+\frac{\mathrm{t}}{\mathrm{T}}\right)$ ou $\mathrm{A}$ et $T$ sont des constantes. En pratique, on était généralement capable de prévoir l'ordre de grandeur de la convergence finale après huit jours d'observation (sinon moins).

\subsection{Les mesures de soulèvement de radier}

Elles ont été faites par nivellement topographique : les résultats des zones les plus déformables sont indiqués à la figure 44 . Les soulèvements se sont réduits à très peu de chose après le bétonnage du béton de roulage. 


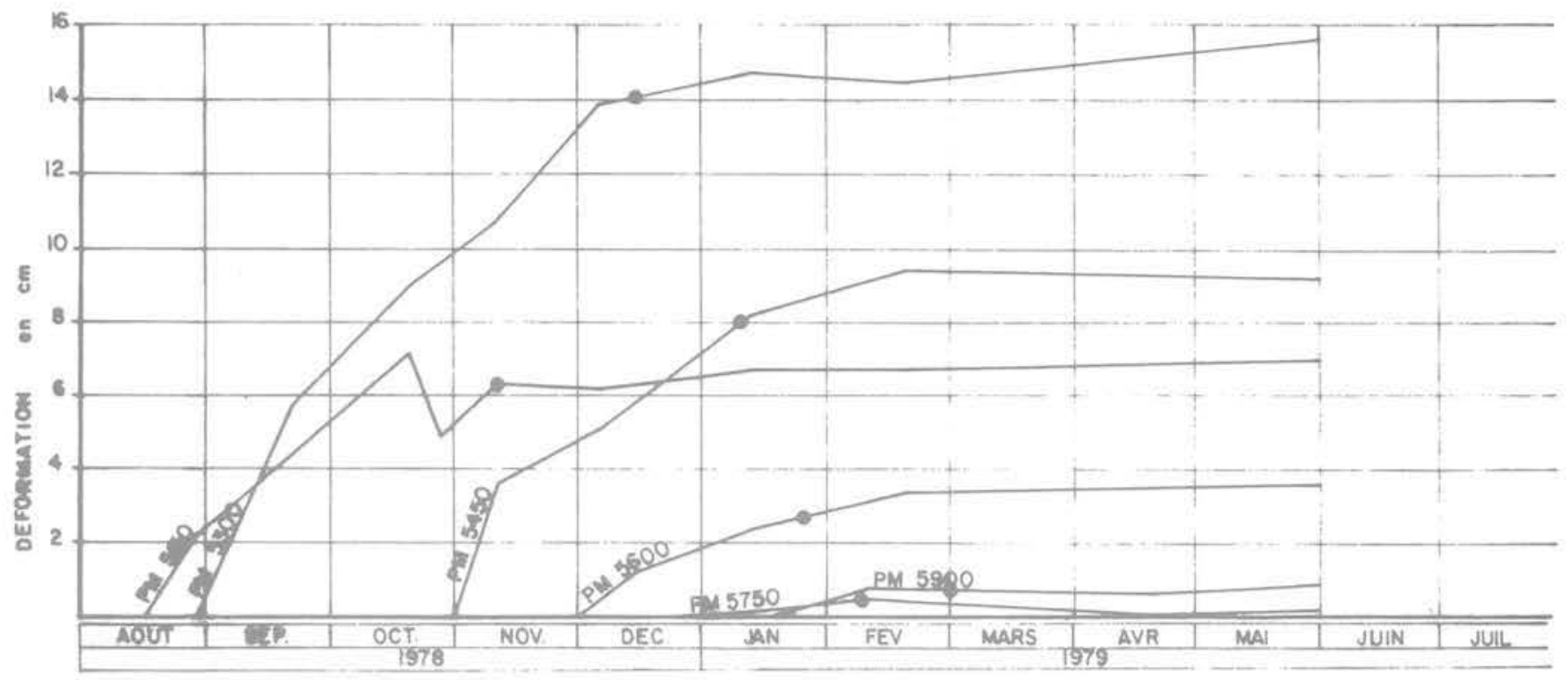

(e Date de betonnage complet du radiar)

PM 6050-6200-6350-6500, deformation nulle

Fig. 44 Soulèvement du radier

\subsection{Les mesures de convergence sur excavation revêtue}

Des bases de mesures de convergence sur revêtement ont été mises en place sur toute la partie française du tunnel. Au total, une trentaine de sections ont été équipées dont 20 dans les schistes lustrés à forte couverture. Les plots $n^{\circ 3} 2$ et 3 ont été abaissés pour être placés juste sous la dalle du plafond. Il est encore trop tôt pour en analyser les résultats pour les raisons suivantes :

- les phénomènes thermiques liés, dans un premier temps, à l'échauffement de prise du béton (puis à son retrait) et, dans un deuxième temps au refroidissement du tunnel après percement sous l'effet de la ventilation naturelle, masquent les effets de la déformation du terrain;

- certains plots ont été endommagés au cours des opérations de chantier puis remis en place.

Cependant, on a d'ores et déjà observé, malgré un net ralentissement d'ensemble des déformations, une convergence de $5 \mathrm{~mm}$ en 8 mois à la section 122 (PM 5205).

\subsection{Les mesures d'expansion sur excavation non revêtue}

Ces mesures ont pour but de mettre en évidence et d'évaluer quantitativement la décompression du terrain qui suit l'excavation du tunnel. Dans le contexte du tunnel du Fréjus, le soutènement provisoire étant constitué de boulons à ancrage ponctuel de $4,65 \mathrm{~m}$ de longueur, on a voulu principalement contrôler si l'expansion des terrains était compatible avec les capacités d'allongement de l'ensemble boulonplaques de répartition.

\subsubsection{Les appareils de mesure}

Trois dispositifs de mesure ont été successivement et simultanément utilisés.

\section{(I) L'appareil-tritige}

Le mouvement d'un point à l'intérieur du massif est transmis à la surface par l'intermédiaire d'une tige d'acier scellée au fond d'un forage.

La mesure du déplacement relatif de l'extrémité de la tige et d'une plaque solidaire de la surface se fait à l'aide d'un comparateur (fig. 45).

Le système est dit tritige car, on exécute trois trous parallèles de profondeurs différentes et on mesure les déplacements par rapport à une même plaque de référence, scellée au parement; les distances sur lesquelles on a mesuré l'expansion étaient de $2 \mathrm{~m}, 4 \mathrm{~m}$ et $6 \mathrm{~m}$ ou de $2 \mathrm{~m}, 5 \mathrm{~m}$ et $10 \mathrm{~m}$.

Fig. 45 Dilatomètre de toit ST 60

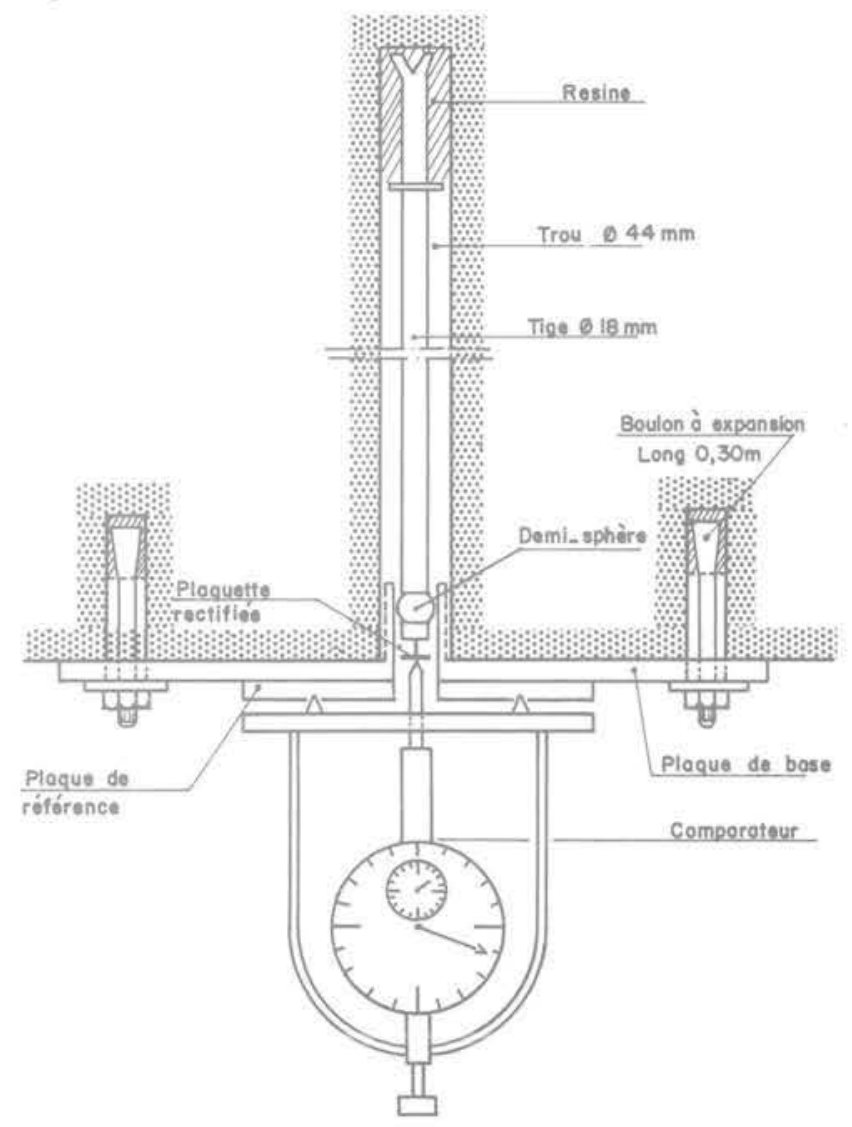


(II) Appareil Distofor (TELEMAC)

Cet appareil décrit par ailleurs, permet en n'utilisant qu'un seul forage, de mesurer l'expansion des terrains à plusieurs profondeurs et de faire cette mesure à distance, ce qui constitue un avantage pratique certain dans le cas d'un tunnel à grande section.

\section{(III) Mesure de convergence différentielle}

Comme pour l'appareil tritige, le mouvement du point situé en profondeur est ramené à la surface par l'intermédiaire d'une tige d'acier scellée en fond de forage à l'extrémité libre de laquelle on fixe un plot de mesure de convergence, un autre plot étant scellé en surface à proximité du premier, il suffit de faire la différence entre les convergences mesurées par rapport au point diamétralement opposé pour obtenir le mouvement relatif du point profond par rapport à la surface, c'est-à-dire l'expansion recherchée.

L'intérêt de ce dispositif de mesure réside dans le fait qu'il ne nécessite pas d'autre appareillage que celui qui est utilisé pour la mesure de la convergence.

\subsubsection{Résultats des mesures}

Ces mesures ont été effectuées aux points métriques suivants :

1976 tritiges

2295 tritiges et Distofor

2954 tritiges et Distofor

4682 convergence différentielle.

5000 à 6000 : une mesure a été faite environ tous les $100 \mathrm{~m}$ par la méthode des convergences différentielles.

Les premières sections de mesures étaient conçues pour mettre en évidence l'expansion parallèlement et perpendiculairement à la schistosité : cinq appareils étaient implantés en éventail au PM 1976 et trois appareils auscultant la voûte dans la section $10 \mathrm{~h}-2 \mathrm{~h}$ étaient implantés au PM 2295.

Ces mesures ont montré une dissymétrie importante des expansions, l'expansion dans la direction perpendiculaire à la schistosité étant très supérieure à l'expansion dans la direction parallèle à la schistosité. Ces expansions étaient dans un rapport de 15 à 1 au PM 1976 et de 100 à 1 au PM 2295 (fig. 46 et 47).

Pour les sections suivantes, la mesure n'a été effectuée que dans la direction perpendiculaire à la schistosité; pour cette direction, l'examen des courbes (fig. 47 et 48) montre que la détente affecte principalement les 5 premiers mètres de la roche; si on considère en effet les $10 \mathrm{~m}$ auscultés au PM 2295 (fig. 47), on constate qu'entre 5 et $10 \mathrm{~m}$ de profondeur, l'expansion ne représente que $15 \%$ de l'expansion totale entre 0 et $10 \mathrm{~m}$. L'évolution de cette expansion se fait à vitesse décroissante et peut comme la convergence, être décrite avec précision par une loi logarithmique tant que le revêtement définitif n'est pas mis en place.

\subsubsection{Effet de l'expansion sur les boulons du soutènement provisoire}

Les mesures d'expansion ont montré que la mise en tension des boulons intervenait dans les premiers jours qui suivaient leur pose; pratiquement, au bout d'un mois, ils avaient subi près de $80 \%$ de l'allongement qu'ils ont au moment où est posé le revêtement définitif, c'est-à-dire 3 à 4 mois après leur pose.

Le tableau ci-contre indiquant les expansions mesurées sur $4,5 \mathrm{~m}$ perpendiculairement à la schistosité au bout de 1 à 2 mois, met bien en évidence la réduction

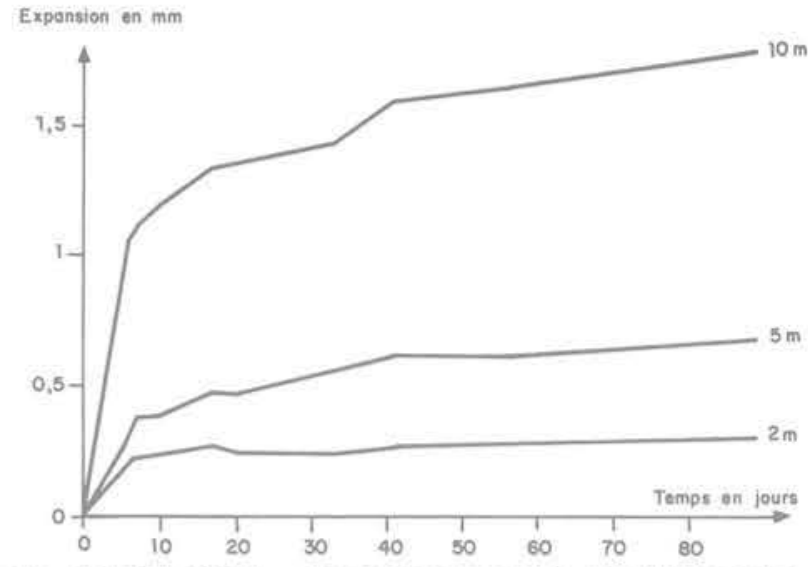

Fig. 46 PM 2295 - Expansion mesurée parallèlement à la schistosité

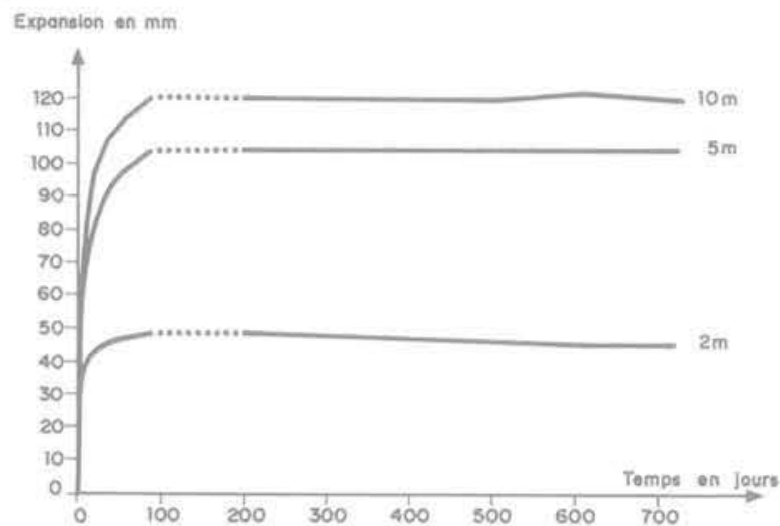

Fig. 47 PM 2295 - Expansion mesurée perpendiculairement à la schistosité

Expansion on $\mathrm{mm}$

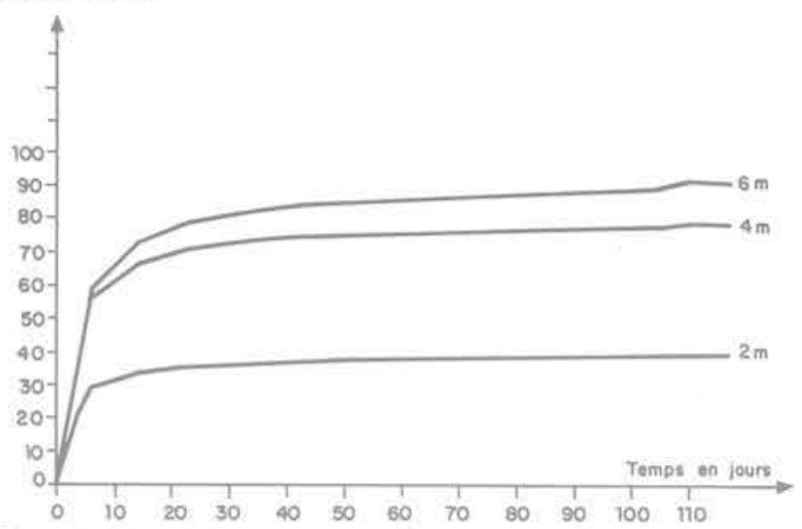

Fig. 48 PM 1976 - Expansion mesurée perpendiculairement à la schistosité

\begin{tabular}{c|c|c}
\hline PM : & $\begin{array}{c}\text { Expansion à 1 mois : } \\
\text { en mm }\end{array}$ & $\begin{array}{c}\text { Expansion à 2 mois : } \\
\text { en mm }\end{array}$ \\
\hline 1976 & 75 & 80 \\
2295 & 89 & 98 \\
2954 & 8 & 11 \\
4681 & 38 & 38 \\
5042 & 118 & 139 \\
5130 & 13 & 13 \\
5204 & 324 & 378 \\
5279 & 82 & 88 \\
5385 & 88 & 103 \\
5462 & 78 & 83 \\
5522 & 78 & 83 \\
5669 & 40 & 45 \\
5742 & 25 & 28 \\
5868 & 12 & \\
5927 & 2 & \\
\hline
\end{tabular}


des vitesses de déformation liée au boulonnage. On constate en effet qu'au cours du $2^{\mathrm{e}}$ mois l'expansion n'augmente plus que de 10 à $15 \%$ quelle que soit son amplitude.

Ces résultats doivent être néanmoins examinés avec une certaine réserve dans la mesure où il est possible que la tige profonde soit pincée (malgré le tubage) par les bancs de schiste glissant les uns par rapport aux autres et que l'ancrage réel de cette tige soit ramené plus près de la surface. Cependant, la conclusion précédente est confirmée par le fait qu'on ne constate plus de rupture de boulons ou d'évolution de la déformation des plaques après le premier mois qui suit leur pose.

\subsection{Les mesures d'expansion en zone revêtue}

Deux stations de mesure au PM 2295 et au PM 2954 ont été maintenues en état après la pose du revêtement définitif. Elles permettent de voir que ce revêtement stoppe presque totalement les expansions, on observe même une recompression du terrain situé au voisinage immédiat de l'anneau de béton (fig. 47).

La station du PM 2295 ayant du être démontée au moment du coffrage du revêtement, il n'y a pas de raccordement entre les parties avant et après bétonnage, en revanche, au PM 2954, la mesure par Distofor a permis de suivre l'évolution de l'expansion pendant toutes les phases des travaux, on observe même une recompression au moment du bétonnage (fig. 49) probablement due à la pression contre le terrain du béton soutenu par le coffrage.

\subsection{Les mesures sur boulons et plaques}

Pour bien comprendre les déformations des plaques de répartition observées et pouvoir interpréter les éventuelles ruptures de boulons, on a établi sur le chantier les courbes de la figure 50 . La courbe 1 donne la déformation du boulon; elle intègre le déplacement éventuel de l'ancrage à coquilles mais s'abstrait des déformations locales du parement grâce à une large plaque d'about rigide en tôle et béton. Sur la tige récupérée après essais, on constate que l'allongement propre de la tige à rupture est de $20 \mathrm{~cm}$ environ.

Les courbes 2 et 3 concernent les plaques de répartition; les boulons les plus sollicités s'appuient en effet sur le terrain par l'intermédiaire d'une large plaque ondulée de $450 \times 650 \times 5 \mathrm{~mm}$ et d'une plaque à rotule $200 \times 200 \times 6 \mathrm{~mm}$.

La courbe 3 représente la déformation d'une plaque à rotule appuyée sur un support rigide (béton).

La courbe 2 représente la déformation globale des deux plaques superposées (grande plaque + plaque à rotule) sur un support rigide.

On constate que l'écrasement complet de la rotule se produit aux environs de la limite élastique du boulon.

On constate, par d'autres essais, que lorsque le parement n'est pas plat (cas fréquent) la grande plaque commence à se déformer plastiquement vers 6 à 8 tonnes.

On voit donc, que grâce au système de soutènement par boulons, plaques et grillage qui laisse les têtes de boulons et les plaques visibles, on dispose de très bons indicateurs pour connaître un ordre de grandeur de la tension qui règne dans les boulons, à chaque instant, et pouvoir intervenir si nécessaire, pour renforcer une zone insuffisamment soutenue.

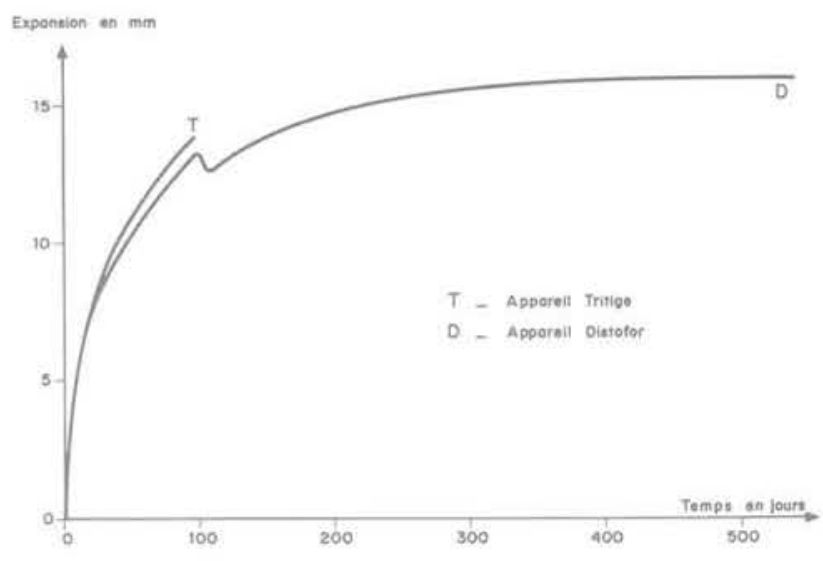

Fig. 49 PM 2954 - Expansion mesurée sur $10 \mathrm{~m}$ perpendiculairement à la schistosité

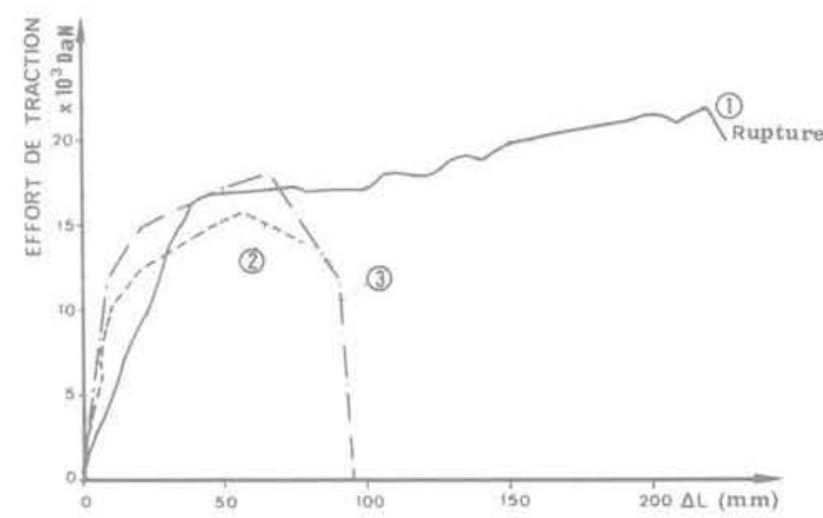

Fig. 50

1 Diagramme de traction au vérin du boulon à ancrage ponctuel : $\emptyset 20,6 \mathrm{~mm} ; \mathrm{l}=4,65 \mathrm{~m}$

2 Poinçonnement de l'ensemble : plaque + rotule (plaque écrasée, rotule déformée)

3 Poinçonnement d'une rotule $200 \times 5$ (rotule écrasée)

Par ailleurs, de nombreux essais de traction sur boulons en place ont été effectués; leur but essentiel était de vérifier la qualité du rocher dans la zone de l'ancrage ponctuel; accessoirement, cela permettait de vérifier la qualité et l'allongement à rupture des boulons.

\subsection{Observations à l'endoscope}

Des observations à l'endoscope pouvant aller jusqu'à $5 \mathrm{~m}$ de profondeur (et exceptionnellement jusqu'à $10 \mathrm{~m}$ ) ont été faites régulièrement dans des trous radiaux généralement perpendiculaires au plan de schistosité; ils servaient à examiner l'état de fracturation ou de fissuration de la roche, en fonction de la profondeur, et son évolution avec le temps.

La figure 51 montre un exemple de relevé dans une zone qui venait de subir une convergence de $40 \mathrm{~cm}$. On constate que la roche est complètement désorganisée jusqu'à $1,40 \mathrm{~m}$ de profondeur par des fissures allant jusqu'à 1 ou $2 \mathrm{~cm}$ et suivant plusieurs directions. Au-delà, les fissures s'amenuisent et s'espacent, la dernière observée étant fine et à une profondeur de $3,60 \mathrm{~m}$. Cela nous permet de vérifier que l'ancrage à coquille des boulons qui est à $4,65 \mathrm{~m}$ est sans doute situé au-delà de la zone fissurée et qu'il est certainement dans une zone résistante où les fissures sont fines et très espacées. 
ETAT DU TERRAIN

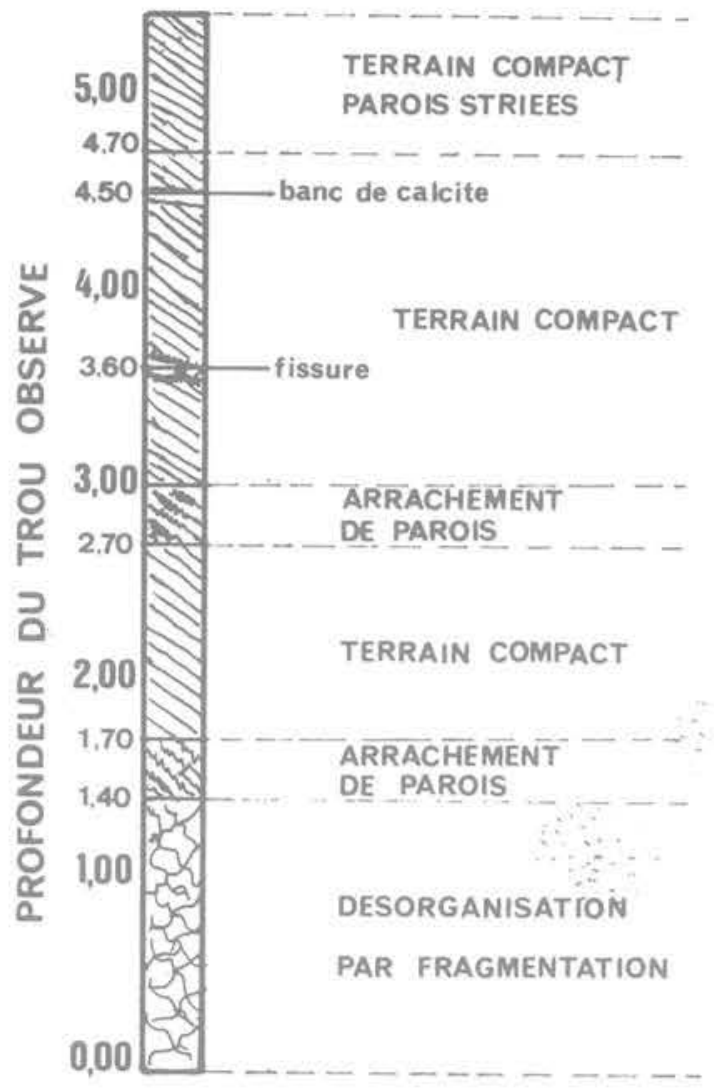

Fig. 51 Observations à l'endoscope

L'observation de l'évolution dans le temps est possible dans les zones peu déformables, ce qui n'est pas très intéressant; elle est généralement impossible dans les zones à grandes déformations qui s'accompagnent d'un glissement des bancs les uns par rapport aux autres ce qui a pour effet d'obturer le trou; on est donc obligé de repercer un trou dans la même zone, à chaque observation.

\section{Mise au point du soutènement provisoire dans les schistes lustrés}

Dans la zone des schistes lustrés rencontrée avant le PM 1710, c'est-à-dire entre les PM 500 et 1480 environ, le soutènement était constitué de boulons à ancrage ponctuel (coquilles d'expansion) de 3 à $4 \mathrm{~m}$ de Iongueur, tige de $18 \mathrm{~mm}$ de diamètre, en acier A70-2 (charge de rupture 190 à $200 \mathrm{KN}$, limite élastique 110 à $120 \mathrm{KN}$ ).

Ces boulons avaient une capacité d'allongement pratique de 3 à $4 \%$. Ils étaient en fait utilisés pour assurer la sécurité du personnel vis-à-vis des chutes de blocs isolés plutôt que pour assurer la stabilité d'ensemble de l'excavation; il est vrai que la couverture était faible $(400$ à $700 \mathrm{~m}$ ) que la résistance du schiste était largement supérieure à la pression des terrains susjacents et que la plupart des boulons paraissaient peu sollicités (pas de déformation des plaques de répartition).

Après le passage d'anhydrite saine du PM 1480 à $1710 \mathrm{~m}$ (qui ne demandait presqu'aucun soutènement), lorsque l'excavation retrouva les schistes lustrés on remit tout naturellement le soutènement qui avait donné satisfaction dans les zones précédentes.
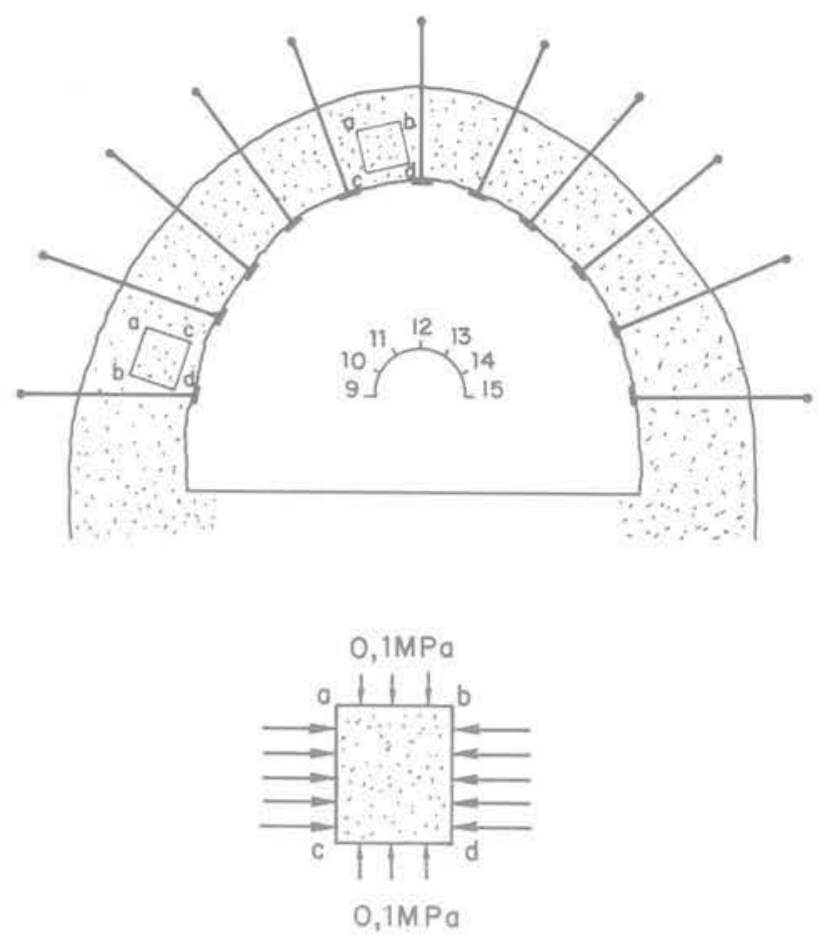

Fig. 52 Efforts exercés par le soutènement

C'est alors qu'on se mit à constater :

- un grand nombre de boulons en charge (plaques déformées), dont certains se rompaient;

- quelques écaillages en piédroits.

Les mesures de convergence effectuées dans cette zone montraient des déformations assez importantes.

C'est sur la base de ces premières observations que fut mis au point le mode de soutènement dans les schistes lustrés déformables, en adaptant le boulonnage suivant les principes que nous allons rappeler et qui ont fait leurs preuves, depuis de nombreuses années, dans les mines métalliques (masses rocheuses relativement résistantes).

\subsection{Rappel du principe de soutènement dans les roches}

Lorsque le front d'une excavation avance, les contraintes tangentielles de la roche en périphérie de l'excavation augmentent jusqu'à atteindre environ le double de la pression moyenne du terrain avant excavation.

Si ces contraintes restent inférieures à la résistance à rupture de la roche, le soutènement peut se réduire à un boulonnage de précaution pour éviter les chutes de blocs éventuellement découpés par la fissuration naturelle ou par les ébranlements dûs aux tirs. Une longueur de boulons égale à $1 / 3$ du diamètre de l'excavation suffit amplement et tout type d'acier peut convenir, notamment l'acier dur qui permet de réduire le diamètre et le poids des boulons pour une même résistance.

Si les contraintes en périphérie dépassent la résistance à rupture de la roche, on voit se former avec l'avancement du front et avec le temps, une zone fracturée de plus en plus épaisse sur tout le pourtour ou sur une partie de l'excavation. Dans cette zone fracturée, le volume de la roche augmente et l'on observe une déformation radiale plus ou moins importante de l'excavation. 
Suivant la théorie classique de la butée, l'application d'une contrainte de compression de 0,1 à $0,2 \mathrm{MPa}$ sur les faces ab et cd d'un élément de roche abcd (fig. 52) lui permet de résister à une contrainte de compression de 2 à $4 \mathrm{MPa}$ sur les faces ac et bd.

On voit donc que si, à l'aide d'un soutènement, on arrive à « maintenir en l'état » et à confiner avec une contrainte de l'ordre de $0,1 \mathrm{Mpa}$ le pourtour de l'excavation, on va créer une sorte d'anneau résistant et déformable en roche fissurée, capable de résister à une charge beaucoup plus élevée que $0,1 \mathrm{MPa}$. L'augmentation de l'épaisseur de la zone fracturée et l'augmentation de volume qui en résulte crée, dans cet anneau, des contraintes radiales suffisantes pour empêcher la fracturation de la roche située au-delà. Pour arriver à cet état d'équilibre, le problème est d'avoir un soutènement qui maintienne un confinement de 0,1 MPa malgré la dislocation croissante de la roche de surface et malgré la déformation de lá périphérie.

Si on utilise des boulons à ancrage ponctuel pour réaliser le soutènement ils doivent répondre aux conditions suivantes :

- avoir un ancrage solide ce qui suppose une longueur de boulon telle que l'ancrage soit situé dans la roche saine au-delà de la zone fracturée et disloquée;

- s'allonger sans se casser malgré les déformations inéluctables de la zone fracturée; cela suppose une longueur de tige suffisante jointe à une qualité d'acier suffisamment déformable.

Par ailleurs, il faut que la roche fracturée ne puisse s'échapper entre les boulons; cela est obtenu en disposant de larges plaques de répartition sous les têtes de boulons et un grillage résistant.

Enfin, il faut un diamètre de tige et une densité de boulons suffisants pour appliquer un confinement de 0,1 à $0,2 \mathrm{MPa}$. Dès l'instant que l'ancrage est au-delà de la zone fissurée, l'expansion des terrains met les boulons en tension; pour miter la déformation on a toutefois intérêt à mettre en place les boulons le plus tôt possible.

\subsection{Choix du soutènement adapté aux schistes lustrés déformables}

\subsubsection{Récapitulation des observations faites sur les 200 premiers mètres de schistes déformables}

L'observation des 200 premiers mètres de schistes lustrés déformables et soutenus avec des boulons courts en acier dur avait donné les résultats suivants :

- il y avait formation d'une zone fracturée surtout autour de $13 \mathrm{~h}$ où le plan de schistosité tangente la voûte, cette zone s'étendant jusque dans le piédroit Ouest (côté droit);

- l'épaisseur des bancs était relativement faible (5 à $15 \mathrm{~cm})$;

- les mesures de convergence donnaient des déformations importantes surtout dans la direction 2-4 transversale au plan de schistosité : $270 \mathrm{~mm}$ au bout d'un mois pour la déformation maximale qui devait atteindre $330 \mathrm{~mm}$ au bout de 4 mois (soit $3 \%$ de diamètre de l'excavation); les courbes de déformations se stabilisaient normalement bien que lentement avec le temps;

- les examens endoscopiques pratiqués à $13 \mathrm{~h}$ dans des trous perpendiculaires à la schistosité révélaient une zone fracturée et même disloquée de $1,5 \mathrm{~m}$ allant jusqu'à 2,5 m (soit $20 \%$ du diamètre de l'excavation); au-delà, les bancs intacts présentaient quelques décollements s'atténuant jusqu'à $3,8 \mathrm{~m}$ de profondeur;

- la résistance de la roche en profondeur permettait l'emploi d'ancrages ponctuels comme le démontraient les ruptures assez nombreuses des boulons au voisinage de l'intrados et comme devaient le confirmer les essais d'arrachement.

\subsubsection{Choix du soutènement à ancrage ponctuel}

Toutes ces observations ont conduit à penser que le soutènement le mieux adapté demeurait le soutènement par boulons à ancrage ponctuel, les plus commodes et les plus rapides à mettre en place avec le matériel présent sur le chantier, à condition de les adapter à la situation constatée

\section{- Longueur :}

La longueur des trous susceptibles d'être forés sans reprise par des glissières inscriptibles dans la section, conduisait à des boulons de $4,65 \mathrm{~m}$, ce qui permettait de placer les ancrages dans la roche saine ou à tout le moins à fissures fines et espacées.

\section{- Qualité d'acier:}

En remplaçant l'acier A 70-2 par l'acier Wemat (limite élastique $450 \mathrm{MPa}$ rupture $=650$ à $800 \mathrm{MPa}, \mathrm{A}=23 \%$ ) on pouvait espérer un allongement pratique de 5 à $6 \%$ sur boulon à rupture soit $250 \mathrm{~mm}$ environ. En ajoutant une plaque de répartition et une plaque à rotule dont la déformation après écrasement était de $100 \mathrm{~mm}$, on obtenait une capacité globale de déformation de $350 \mathrm{~mm}$.

Cela paraissait largement suffisant puisque la convergence maximale mesurée était de $330 \mathrm{~mm}$ et que l'expansion du point 2 sur les 4,65 premiers mètres n'est qu'une partie de la convergence globale sur le diamètre 2-4 (il faut en retirer le mouvement faible mais non nul du point 4 et la déformation des couches situées au-delà de 4,65 m dans la direction du point 2).

\section{- Densité de boulonnage :}

Dans la zone la plus sensible, la densité de boulonnage atteignait 1,2 boulons par $\mathrm{m}^{2}$. Le diamètre étant passé de 18 à $20 \mathrm{~mm}$ pour compenser l'adoucissement de l'acier, cela conduisait à un confinement de 0,18 MPa à la limite élastique, montant jusqu'à plus de 0,25 MPa si I'on devait se rapprocher de la limite de rupture des boulons.

Notons que la tige de boulon atteint sa limite élastique dès qu'on l'allonge de $2 \cdot 10^{-3}$ soit $1 \mathrm{~cm}$ ce qui se produit très peu de temps après mise en place du soutènement.

- Plaques:

Après utilisation temporaire de plaques d'envol $3000 \times 400 \times 3 \mathrm{~mm}$, on utilisa assez rapidement des plaques de répartition $650 \times 450 \times 5 \mathrm{~mm}$. Les plaques à rotules avaient des dimensions de $200 \times 200 \times 6 \mathrm{~mm}$.

- Grillage :

Pour permettre un confinement au cas où la roche fracturée s'échapperait entre plaques, ainsi que pour résister aux tirs, en étant mis à front, on choisit un grillage à fil de $5 \mathrm{~mm}$ et mailles de $10 \mathrm{~cm}$; de toute façon, ce grillage servait aussi de filet de protection indispensable en raison de la hauteur de la section. 


\subsection{Adaptation du soutènement sur le reste du tunnel}

Après la mise au point précédente, on constata une amélioration nette :

- disparition des ruptures des boulons:

- diminution des convergences.

Cependant après 2500 m d'avancement, on arriva dans une zone plus fracturée, plus riche en graphite et plus déformable. C'est ainsi qu'on atteignit, à la section $114,370 \mathrm{~mm}$ à un mois, $463 \mathrm{~mm}$ à 3 mois et $500 \mathrm{~mm}$ à 4 mois.

Dans cette zone on nota à nouveau quelques ruptures de boulons (sans dépasser 2 boulons par $10 \mathrm{~m}$ de tunnel).

En prévision d'une aggravation de la situation on modifia le soutènement de la façon suivante:

- changement de qualité d'acier : acier A 50 ( $E=$ $280 \mathrm{MPa} ; \mathrm{R}=500 \mathrm{MPa}$ );

- augmentation du diamètre : $24 \mathrm{~mm}$ au lieu de 20 pour compenser l'adoucissement de l'acier et augmenter le confinement (jusqu'à $0,3 \mathrm{MPa}$ ) car on ne peut augmenter exagérément la densité de boulonnage sans affaiblir le terrain.

On fut amené à utiliser également par endroits :

- des boulons Ø25 mm de 5,30 m en acier Alpha 4;

- la technique du reboulonnage dans les zones très déformables.

On approvisionna des cales de bois à disposer entre la petite et la grande plaque pour absorber quelques centimètres supplémentaires de déformation mais on n'eut pas finalement à les utiliser.

Enfin, il faut noter que dans quelques zones très faillées, on fut amené à avoir des doutes sur la tenue des ancrages (essais au vérin) et on compléta le soutènement en boulons, par des anneaux en béton, cintrés par des HEB 200 incorporés.

Les observations faites sur le comportement de ces zones de longueur très limitée (trois tronçons d'une dizaine de mètres chacun entre les PM 5100 et 5410) ont confirmé, par ailleurs, que des anneaux bétonnés rigides ne sont pas aptes à supporter les déformations initiales des schistes lustrés du Fréjus.

\subsection{Durée de mise en place du soutènement}

Le soutènement par boulons étant mis en place à front et donc dans le cycle, il est intéressant de noter la durée des opérations. Pour une longueur de volée de $4,5 \mathrm{~m}$ en pleine section, on compte environ 90 à 100 boulons avec leurs plaques à rotules, 60 à 80 grandes plaques de répartition et 2 rouleaux (ou 3 ) de grillage de $2,2 \mathrm{~m} \times 18 \mathrm{~m}$.

La durée moyenne d'un cycle étant de $11 \mathrm{~h} 15$, on compte environ $2 \mathrm{~h} 15$ à $2 \mathrm{~h} 30$ pour le boulonnage et $0 \mathrm{~h} 40$ à $1 \mathrm{~h}$ pour la mise en place du grillage. Ce soutènement était exécuté à l'aide de 2 jumbos Montabert comportant chacun 3 bras et une nacelle et pouvant être mis côte à côte. La durée de mise en place du soutènement représente donc un peu moins du tiers de la durée du cycle.

\section{Revêtement définitif}

\section{1 Étude de l'avant-projet}

L'étude de l'avant-projet fut faite de façon très pragmatique. Le tunnel ferroviaire voisin, âgé de 100 ans a un revêtement en maçonnerie, qui a, dans l'ensemble, convenablement tenu. Des mesures de contraintes au vérin faites par le laboratoire de l'École Polytechnique de Paris et la S.E.I.L mirent en évidence des contraintes assez élevées; cependant une extrapolation de ces résultats conduisit à une épaisseur minimale de $0,35 \mathrm{~m}$ pour le revêtement en béton du tunnel routier du Fréjus, malgré toutes les incertitudes qui résultent de constatations faites sur un tunnel qui a été réalisé en 7 phases successives d'excavation et de revêtement.

\section{2 Étude du projet}

Une autre approche a été tentée pour le projet en utilisant une méthode de calcul aux éléments finis dans le domaine élastoplastique avec le programme ROSALIE du L.C.P.C.

Partant de la constatation que la déformation de la galerie était stabilisée par le soutènement provisoire, on a déterminé la voûte nécessaire pour porter la zone tenue par les boulons après corrosion de ces derniers; on a ainsi appliqué le poids d'une cloche de $4 \mathrm{~m}$ d'épaisseur.

On a pu démontrer qu'un revêtement de $0,35 \mathrm{~m}$ de béton équilibrait facilement une cloche de $4 \mathrm{~m}$ (contrainte de compression inférieure à $3,5 \mathrm{MPa}$ ) et même de $6 \mathrm{~m}(5 \mathrm{MPa})$ et plus.

\subsection{Exécution}

A l'exécution, on a constaté que l'effet des déformations de la zone de roche fracturée à soutenir est prépondérant par rapport à son poids bien que le comportement de la voute, en déformations imposées. soit théoriquement indépendant de son épaisseur.

On s'est donc efforcé de mettre le revêtement après que les déformations se soient stabilisées le plus longuement possible.

L'organisation de chantier y conduisait naturellement, néanmoins on a souvent été amené à bétonner le revêtement à une périodé où la convergence était située entre 0,1 à $0,5 \mathrm{~mm} /$ jour.

On a mis en place les moyens d'observer les déformations du revêtement mais il est encore trop tôt pour pouvoir tirer des conclusions sur le comportement de la voûte revêtue.

\section{Conclusion}

Sur ce chantier, un effort important a été fait pour déterminer le comportement du schiste, de l'excavation et du soutènement (l'exploitation théorique des mesures accumulées doit d'ailleurs se poursuivre). Cet 
effort s'est étendu à la surveillance de la stabilité de l'excavation non revêtue et à la mise au point d'indicateurs fiables d'alerte. Les importantes dépenses engagées à cet effet ont eu au moins les contreparties suivantes :

- jamais le soutènement provisoire n'a été pris en défaut tout au long des $4,5 \mathrm{~km}$ d'excavation dans les schistes lustrés déformables et on a toujours pu intervenir à temps pour compléter le soutènement ou le modifier en cas de besoin, tout en réduisant à une faible longueur les zones à soutènement spécial;

- la connaissance des phénomènes due à cette surveillance serrée et permanente, la confiance qui en résultait dans les soutènements mis en place, ont permis d'adopter, sans perte de temps, les mesures appropriées dans les zones les plus déformables et de maintenir une cadence d'avancement exceptionnellement élevée et régulière sur l'ensemble du chantier d'excavation.

Tous les partenaires de ce chantier en retirent l'impression que les économies ainsi réalisées compensent largement les dépenses engagées pour les mesures géotechniques dont il faut rappeler, malgré tout, que leur objectif essentiel était d'assurer sur le chantier une sécurité maximale des personnels. 